\title{
Retinoic-acid-orphan-receptor-C inhibition suppresses Th17 cells and induces thymic aberrations
}

\author{
Christine Guntermann, ${ }^{1}$ Alessandro Piaia, ${ }^{2}$ Marie-Laure Hamel, ${ }^{3}$ Diethilde Theil, ${ }^{2}$ \\ Tina Rubic-Schneider, ${ }^{2}$ Alberto del Rio-Espinola, ${ }^{2}$ Linda Dong, ${ }^{4}$ Andreas Billich, ${ }^{1}$ \\ Klemens Kaupmann, ${ }^{1}$ Janet Dawson, ${ }^{1}$ Klemens Hoegenauer, ${ }^{5}$ David Orain, ${ }^{5}$ Samuel Hintermann, ${ }^{5}$ \\ Rowan Stringer, ${ }^{6}$ Dhavalkumar D. Patel, ${ }^{1}$ Arno Doelemeyer, ${ }^{2}$ Mark Deurinck, ${ }^{2}$ and Jens Schümann ${ }^{2}$ \\ 'Autoimmunity, Transplantation, and Inflammation Disease Area, ${ }^{2}$ Preclinical Safety, Novartis Institutes for BioMedical \\ Research, Basel, Switzerland. ${ }^{3}$ CiToxLAB, Evreux, France. ${ }^{4}$ Preclinical Safety, Novartis Institutes for BioMedical Research, \\ East Hanover, New Jersey, USA. ${ }^{5}$ Clobal Discovery Chemistry, ${ }^{6}$ Metabolism and Pharmacokinetics, Novartis Institutes for \\ BioMedical Research, Basel, Switzerland.
}

Retinoic-acid-orphan-receptor-C (RORC) is a master regulator of Th17 cells, which are pathogenic in several autoimmune diseases. Genetic Rorc deficiency in mice, while preventing autoimmunity, causes early lethality due to metastatic thymic T cell lymphomas. We sought to determine whether pharmacological RORC inhibition could be an effective and safe therapy for autoimmune diseases by evaluating its effects on Th17 cell functions and intrathymic T cell development. RORC inhibitors effectively inhibited Th17 differentiation and IL-17A production, and delayed-type hypersensitivity reactions. In vitro, RORC inhibitors induced apoptosis, as well as Bc/2/1 and BCL2L1 mRNA downregulation, in mouse and nonhuman primate thymocytes, respectively. Chronic, 13-week RORC inhibitor treatment in rats caused progressive thymic alterations in all analyzed rats similar to those in Rorc-deficient mice prior to T cell lymphoma development. One rat developed thymic cortical hyperplasia with preneoplastic features, including increased mitosis and reduced IKAROS expression, albeit without skewed T cell clonality. In summary, pharmacological inhibition of RORC not only blocks Th17 cell development and related cytokine production, but also recapitulates thymic aberrations seen in Rorc-deficient mice. While RORC inhibition may offer an effective therapeutic principle for Th17-mediated diseases, T cell lymphoma with chronic therapy remains an apparent risk.

Conflict of interest: MLH works for CiToxLAB performing studies by order of Novartis. ADRE works for Novartis. All other authors work for and own shares or options of Novartis.

Submitted: October 11, 2016 Accepted: January 17, 2017 Published: March 9, 2017

Reference information: JCI Insight. 2017;2(5):e91127. https:// doi.org/10.1172/jci.insight.91127.

\section{Introduction}

Retinoic-acid-orphan-receptor-C (RORC), also known as $\mathrm{ROR} \gamma$, is a nuclear hormone receptor playing an important role in immunity, metabolism, and expression of peripheral clock genes. Two isoforms of RORC - $\mathrm{RORC}_{\mathrm{var} 1}$ and $\mathrm{RORC}_{\mathrm{var} 2}$, also known as ROR $\gamma 1$ and $\mathrm{ROR} \gamma 2$ (ROR $\gamma \mathrm{t}$ ) - have been identified. RORC, particularly $\mathrm{RORC}_{\mathrm{var} 2}$, is considered as a drug target because it is a key transcription factor for the differentiation and effector function of the proinflammatory Th17 cells, which drive a number of autoimmune diseases (1-3). Both isoforms differ by addition of the first 21 amino acids in $\mathrm{RORC}_{\mathrm{var}}$ due to different promoter usage. Current low-molecular weight inhibitors target the identical ligand binding domain (LBD) common to both isoforms (2), thus inhibiting the activity of both RORC isoforms.

RORC inhibitors have been reported to block Th17 differentiation in vitro and to inhibit immune reactions in in vivo models of experimental autoimmune encephalomyelitis, imiquimod-induced cutaneous inflammation, and collagen-induced arthritis (2-7). Clinical proof-of-concept trials with the RORC inhibitors VTP43742 (oral; ref. 8) and GSK2981278 (topical; ref. 9) in psoriasis patients are ongoing or have been completed.

RORC is a transcription factor that binds as a monomer to the ROR response elements in the promoter of its target genes. $\mathrm{RORC}_{\mathrm{var}}$ is ubiquitously expressed, whereas $\mathrm{RORC}_{\mathrm{var} 2}$ expression is restricted to leukocytes (e.g., Th17 cells, lymphoid tissue inducer cells [LTi cells], and invariant NK T cells [iNKT cells] ; refs. 1, 10), whose development depends on the expression of this transcription factor. $\mathrm{RORC}_{\mathrm{var} 2}$ is transiently expressed during thymocyte development. Its expression is particularly high when immature single-positive (ISP) $\mathrm{CD}^{+}$ cells differentiate into $\mathrm{CD}^{+} \mathrm{CD} 8^{+}$double-positive (DP) thymocytes (11-13). 
RORC physiological roles have been identified in Rorc-deficient $\left(\right.$ RorC $\left.^{--}\right)$mice and patients $(1,10-12$, 14, 15). Rorc $^{-1-}$ mice are characterized by a lack of lymph nodes, Peyer's patches, and other intestinal lymphoid cell compartments, along with an absence of Th17, LTi, and iNKT cells due to impaired development. $\mathrm{RorC}^{-1}$ mice have a shorter life expectancy, with $50 \%$ of $\mathrm{RorC}^{-1}$ mice dying within 4 months due to metastasizing thymic $\mathrm{T}$ cell lymphomas (14).

The thymi of $\mathrm{Rorc}^{-1-}$ mice show specific aberrations, which occur prior to the development of $\mathrm{T}$ cell lymphoma. These include reduced total (including DP) cell numbers, reduced expression of CD4 on DP cells/increased numbers of $\mathrm{CD}^{+} \mathrm{SP}$ cells, increased frequencies of thymocytes in the S/G2/M cell cycle phase, reduced B cell lymphoma-extra large $\left(B C L-X_{L}\right)$ expression, larger cell size and increased apoptosis in the cortex (where the DP cells reside), and increased cortical/medullary ratio. In vitro, Rorc ${ }^{-1}$ mousederived thymocytes are characterized by undergoing accelerated spontaneous apoptosis.

Recently, it was reported that induction of Rorc deficiency in adult mice also leads to the development of thymic lymphoblastic lymphoma, similar to the thymic T cell lymphoma found in constitutive Rorc ${ }^{\prime-}$ mice and within a similar time frame (16).

$R O R C^{-1-}$ patients display immunological features concordant with $\mathrm{RorC}^{-1-}$ mice, including the absence of palpable axillary and cervical lymph nodes consistent with LTi deficiency, lack of iNKT cells, abolished production of IL-17A/F, and reduced thymus size. Immunity to Mycobacterium and Candida is impaired in these patients, explained by a defective IFN- $\gamma$ response to Mycobacterium and the absence of IL-17A/F, respectively (15)

In this report, we describe the pharmacological characterization of 2 structurally unrelated RORC inhibitors. One of the compounds had favorable PK properties and was used for further in vivo efficacy testing in rats and to assess thymic alterations associated with pharmacological inhibition of RORC in a 13-week safety study.

We demonstrate that targeting RORC by low-molecular weight compounds results in selective blockade of the proinflammatory Th17/IL-17A pathway and shows good efficacy in an in vivo delayed-type hypersensitivity (DTH) model. We report here for the first time to our knowledge that, upon prolonged pharmacological RORC suppression, thymic aberrations occur in rats that are reminiscent to those observed in $\mathrm{Rorc}^{\prime-}$ mice prior to the development of thymic T cell lymphoma. There was one animal that showed advanced signs of aberrations, including thymic cortical hyperplasia with preneoplastic features.

\section{Results}

Identification of potent human RORC inhibitors. Compound 1 ( $\mathrm{cpd} 1$ ) was synthesized as described in a GlaxoSmithKline patent (17). Synthesis of the structurally unrelated RORC inhibitor cpd 2 is described in the Supplemental Material (supplemental material available online with this article; https://doi. org/10.1172/jci.insight.91127DS1). Chemical structures are given in Supplemental Figure 1.

In a time-resolved Foerster resonance energy transfer assay (TR-FRET assay), both compounds inhibited the binding of the human RORC LBD to the RIP140 cofactor peptide with half-maximal inhibition $\left(\mathrm{IC}_{50}\right)$ values of 1.2 and $0.3 \mathrm{nM}$ for cpds 1 and 2, respectively (Figure 1A). This assay relies on the compound-induced disruption of the RIP140 cofactor interaction with the RORC-LBD. Furthermore, in a cellular RORC-LBD/GAL4 reporter gene assay, both compounds potently inhibited RORC-dependent transactivation with $\mathrm{IC}_{50}$ values ranging between 22 and $32 \mathrm{nM}$ (Figure 1B). We next assessed the effect of these compounds on RORA and RORB transcriptional activity in analogous cellular assays. Both compounds were selective for RORC and lacked appreciable activity against RORA (Figure 1C) and RORB (Figure 1D) in GAL4 reporter gene assays.

Selective blockade of primary Th17 and Tc17 cell differentiation by RORC inhibitors. In a primary human Th17 polarization assay, both RORC inhibitors potently inhibited IL-17A secretion in a concentration-dependent manner, with $\mathrm{IC}_{50}$ values of 11 and $36 \mathrm{nM}$ for cpds 1 and 2, respectively (Figure 2A). Likewise, when human naive $\mathrm{CD}^{+} \mathrm{T}$ cells were differentiated under Th17 skewing conditions for 4 days, both compounds potently inhibited Th17 differentiation, as demonstrated by almost complete reduction of IL-17A production (Figure 2B). Following polarization of $\mathrm{CD} 8^{+} \mathrm{T}$ cells toward an IL-17A-producing Tc17 phenotype, the RORC inhibitors affected IL-17A production in these cells, as well. Both compounds were equipotent and led to an almost complete inhibition of IL-17A secretion, with $\mathrm{IC}_{50}$ values between 4 and $10 \mathrm{nM}$ (Figure 2C). No significant cell death was observed at any compound concentrations tested (data not shown), indicating that the observed inhibition of Th17 cell polarization was not due to decreased cell 
A
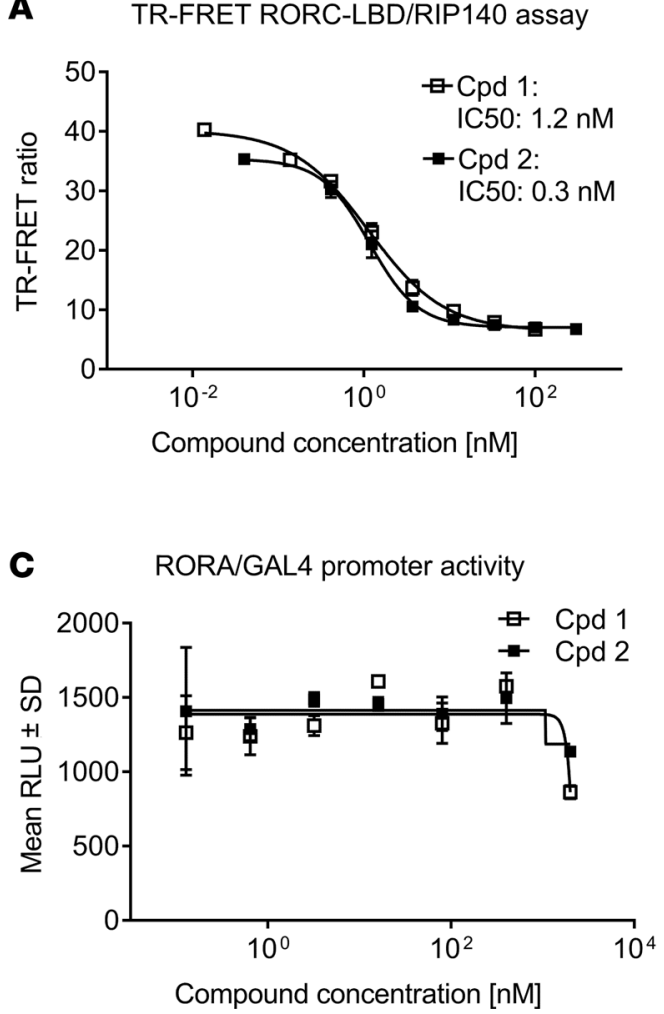

B RORC/GAL4 promoter activity

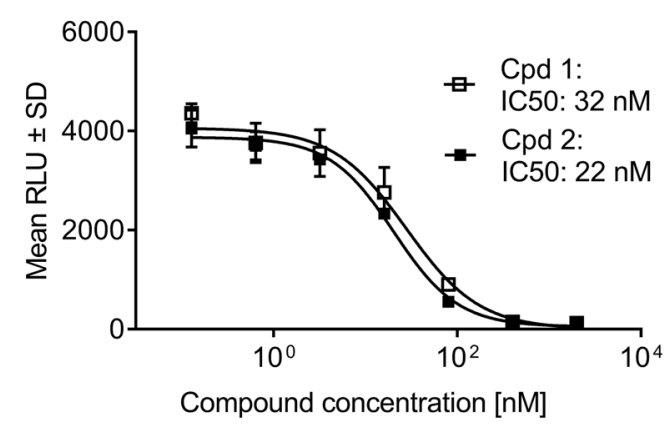

D RORB/GAL4 promoter activity

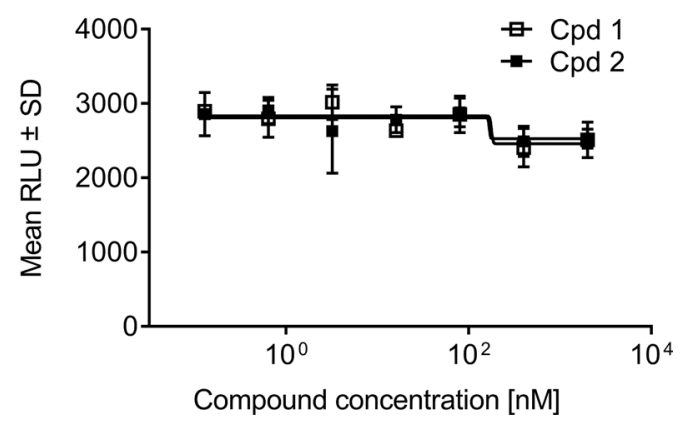

Figure 1. Potency and selectivity of retinoic-acid-orphan-receptor-C (RORC) inhibitors (cpd 1 and cpd 2). (A) Time resolved Foerster resonance energy transfer (TR-FRET) assay demonstrating concentration-dependent inhibition of RIP140 cofactor displacement from the RORC ligand binding domain (RORC-LBD). Data represent means from duplicate readings from at least 3 independent experiments. (B) Cpds 1 and 2 are potent and selective inhibitors of RORC transcriptional activation in a cellular RORC reporter gene assay. Cpds 1 and 2 did not affect RORA (C) or RORB (D) activity in reporter gene assays. Representative concentration-dependent curves from 3 independent experiments containing triplicate readings are shown. Inhibitory concentrations to achieve $50 \%$ of the signal $\left(\mathrm{IC}_{50} \mathrm{~s}\right)$ are indicated in the graphs.

viability/proliferation. The RORC inhibitors acted selectively on the Th17 pathway, since they had no effect on the secretion of the respective signature cytokines IL-2 (Figure 2D), IFN- $\gamma$ (Figure 2E), or IL-13 (Figure 2F) of Th0, Th1, or Th2 cells, respectively. The potency and selectivity of the RORC inhibitors on Th17 development was also evident by intracellular IL-17A staining (Figure 2G). The number of IL-17A cells within the $\mathrm{CD} 4^{+} \mathrm{T}$ cell compartment was diminished by $75 \%$ and $92 \%$ in the presence of cpd 1 and cpd 2, respectively. Neither compound significantly affected the frequencies of IL-2, IFN- $\gamma-$, or IL-4-producing Th0, Th1, or Th2 cells, respectively.

The 2 RORC inhibitors also attenuated the acute expression of the IL17A gene in PMA/ionomycin-stimulated purified human innate $\gamma \delta \mathrm{T}$ cells in a concentration-dependent manner, suppressing by $74 \%$ (cpd 1) or 90\% (cpd 2) within 24 hours (Figure $2 \mathrm{H}$ ). These cells constitutively express RORC and have been implicated in the pathology of psoriasis (18).

In a Th17 polarization assay with rat $\mathrm{T}$ cells, both compounds almost fully inhibited IL-17A production with similar potencies to those observed in human primary Th17 cells (Figure 2I), indicating that the functional role of RORC to potentiate IL-17A production is conserved in both species.

Downregulation of Th17 signature gene expression after pharmacological inhibition of RORC. We next assessed whether expression of Th17 signature genes apart from IL-17A that are directly regulated by RORC (19-21) may also be modulated by cpds 1 and 2 . Human Th17 cells polarized for 3 days in the presence of RORC inhibitors were examined for RORC target gene expression levels by quantitative PCR (qPCR). We found that the compounds reduced Th17 cell-associated mRNA expression of known RORC targets, namely IL17A (Figure 3A), IL17F (Figure 3B), IL22 (Figure 3C), IL26 (Figure 3D), and CCR6 (Figure 3E), both compounds to a similar extent. The expression levels of the RORC target $I L 23 R$ were reduced by $>20 \%$ by the compounds (Figure $3 \mathrm{~F}$ ). Both compounds had no effects on RORC expression levels (Figure 3G), in line with their action as 
A Th17 cell polarization (total CD4 T-cells)

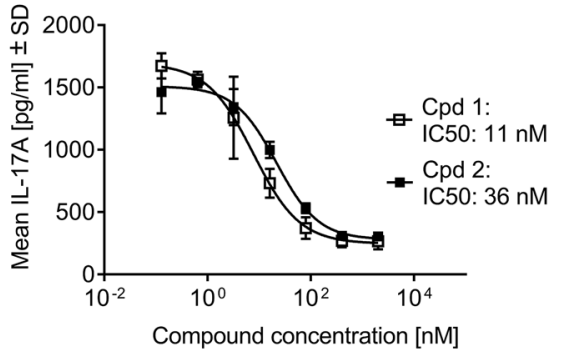

D

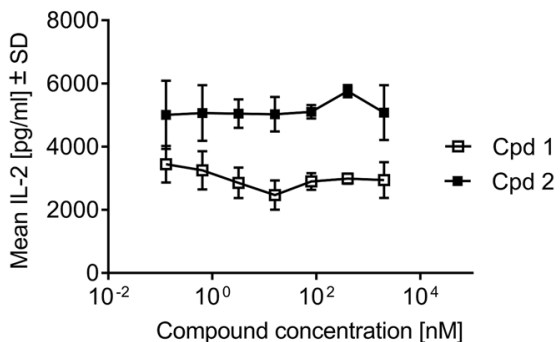

G

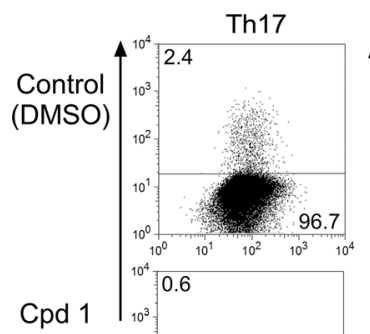

$[1 \mu \mathrm{M}] \ll$

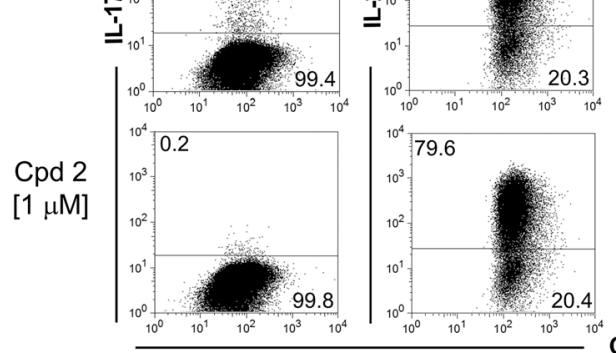

B

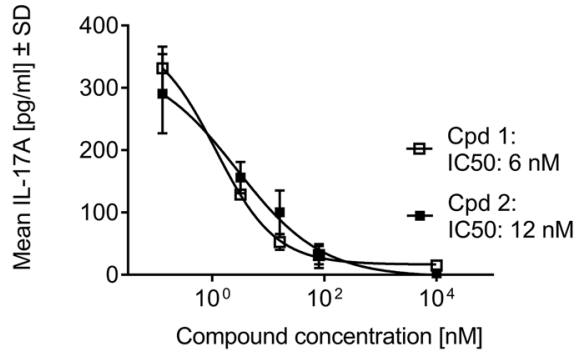

E

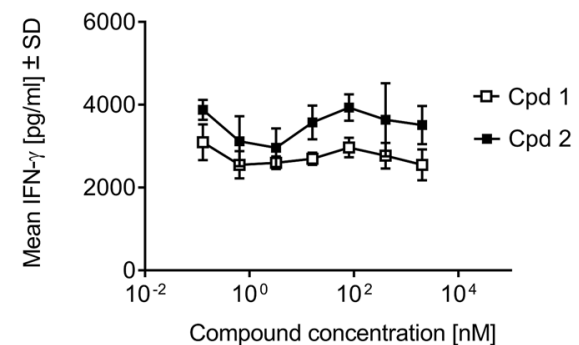

Compound concentration [nM]

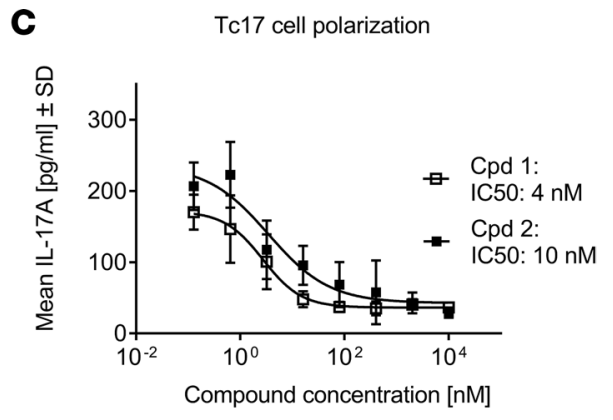

$\mathbf{F}$

Th2 cell polarization

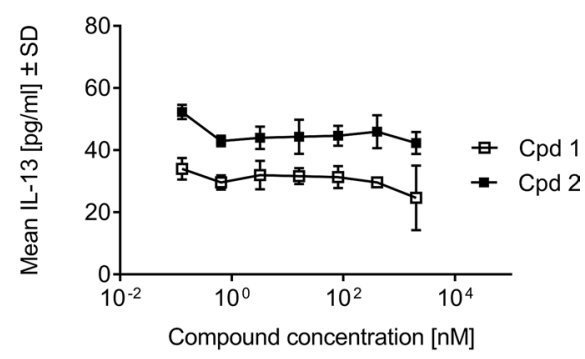

H

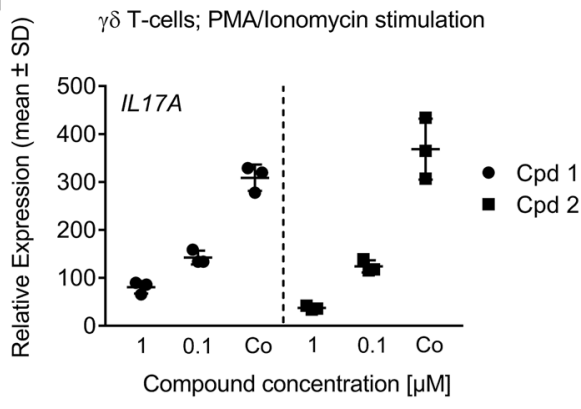

I Rat Th17 cell polarization

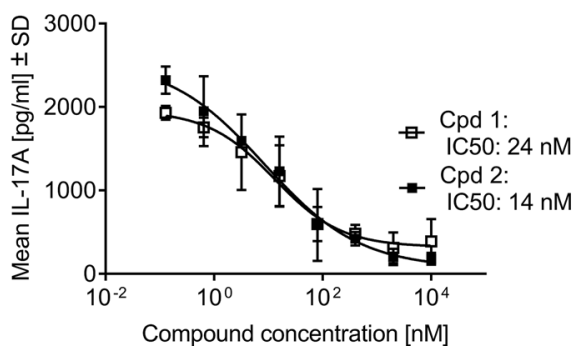

Figure 2. Effects of retinoic-acid-orphan-receptor-C (RORC) inhibitors (cpd 1 and cpd 2) on T cells in vitro. RORC inhibitors (cpd 1 and cpd 2) specifically suppress human Th17 (A-B) or Tc17 cell polarization (C), while leaving Tho (D), Th1 (E), or Th2 (F) signature cytokine production intact. Representative examples of concentration-response curves from 3 experiments with triplicate readings are shown. Total (A) or naive $C D 4^{+}(\mathbf{B})$ or $\mathrm{CD} 8^{+}(\mathbf{C}) \mathrm{T}$ cells derived from blood of human healthy donors were stimulated with anti-CD3 and anti-CD28 antibodies in the presence of Th17-polarizing cytokines for 72 or 96 hours, and IL-17A production was quantified. Alternatively, CD4+ T cells were stimulated with anti-CD3/CD28 only (Th0) (D) and incubated with IL-12 and anti-IL-4 antibody (Th1) (E), or with IL-4 and anti-IFN- $\gamma$ antibody (Th2) (F). IL-2, IFN- $\gamma$, and IL-13 cytokine production by the respective Th subsets was analyzed by ELISA after 48 hours. (C) Human CD4+ $T$ cells were activated under Th17-, Th0-, Th1-, or Th2-polarizing conditions in the presence of RORC inhibitors or DMSO. Production of IL-17A, IL-2, IFN- $\gamma$, or IL-4 was analyzed by intracellular staining and flow cytometry. Intracellular cytokines within $\mathrm{CD}^{+} \mathrm{T}$ cell blasts are shown. Data are representative of 2 independent experiments. (H) Human $\gamma \delta$ T cells were incubated with RORC inhibitors or DMSO only (Co) and stimulated with PMA/ionomycin for 24 hours. IL17A transcript levels were quantified by RT-PCR. Gene expression was normalized to $\beta$-glucuronidase levels and is expressed as arbitrary units. Results are representative of 2 independent experiments. Individual data and mean \pm SD from triplicate readings are depicted. (I) $C D 4^{+} T$ cells isolated from splenocytes from male Lewis rats were stimulated with anti-CD3 and anti-CD28 antibodies in the presence of Th17-polarizing cytokines. IL-17A concentrations in supernatants were determined by ELISA. Representative examples of concentration-response curves from 3 experiments with triplicate readings are shown. 
A

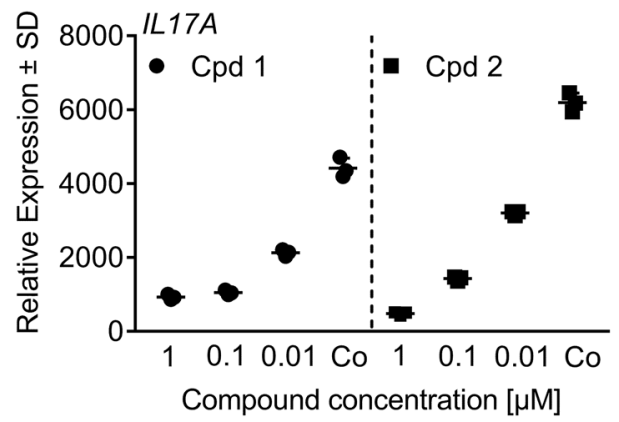

C

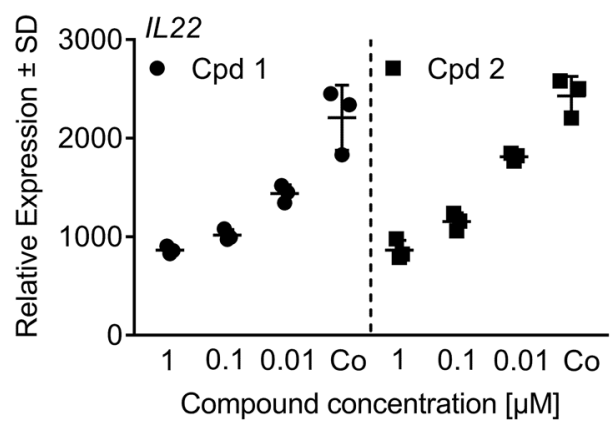

E

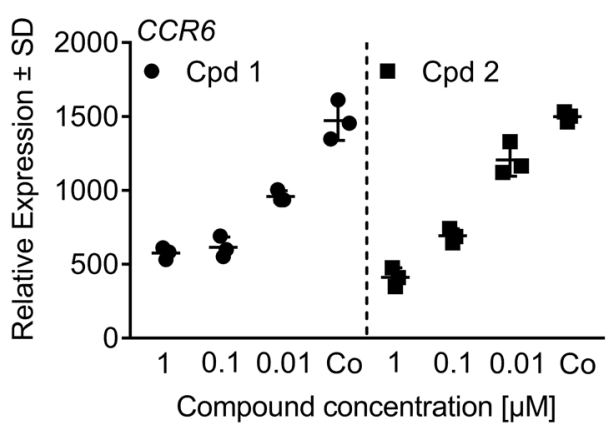

G

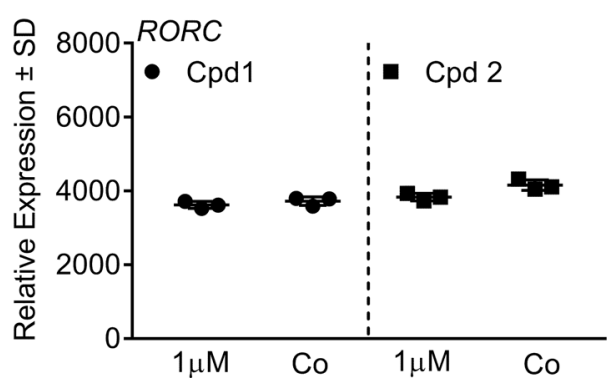

B

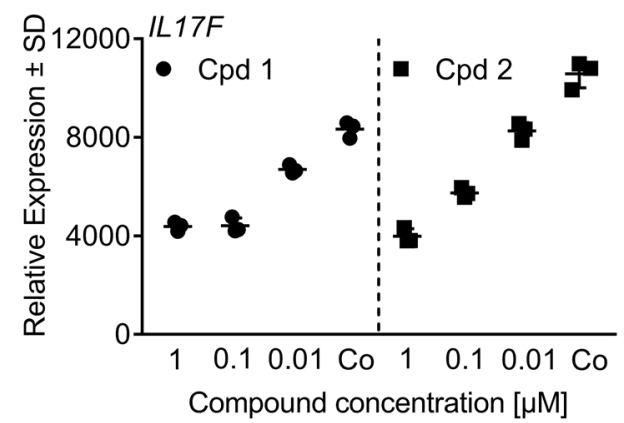

D

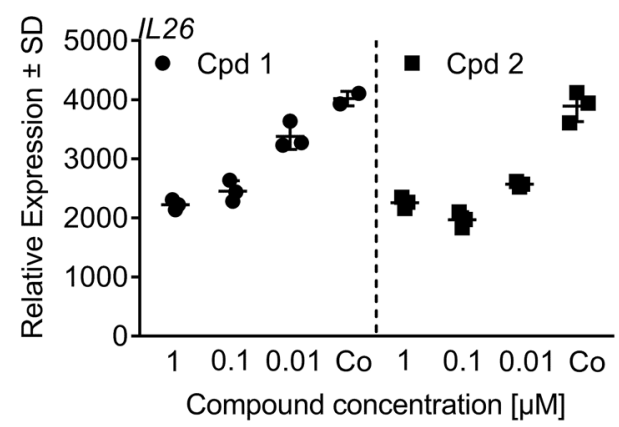

$\mathbf{F}$

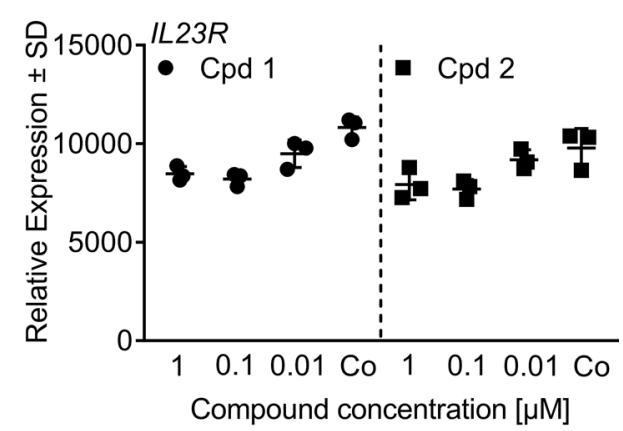

Figure 3. Reduced retinoic-acid-orphan-receptor-C-dependent (RORC-dependent) target gene expression by cpds 1 and 2. CD4 ${ }^{+}$Th17 cells were treated with compounds ( $10 \mathrm{nM}-1 \mu \mathrm{M})$ or with DMSO only (Co) during 72 hours, mRNA was extracted, and transcript levels were quantified by RT-PCR. Gene expression was normalized to $\beta$-glucoronidase levels and expressed as arbitrary units. (A-G) All graphs are representative of 3 independent experiments. Individual data and mean \pm SD from triplicate readings are shown. The DMSO control shown in the cpd 1 panel in $\mathbf{D}$ consisted of 2 readings.

inhibitors of RORC transcriptional activity. The compounds did not affect IFNG levels (data not shown), suggesting that inhibition of RORC did not result in increased propensity of cells to shift toward a Th1 cell phenotype.

In summary, cpds 1 and 2 are potent and selective inhibitors of RORC, repressing the RORC-dependent gene expression program and cytokine production by human and rat Th17 or Tc17 cells.

Physicochemical properties and rat pharmacokinetics. Before testing in vivo efficacy and safety, the physicochemical and pharmacokinetic properties of cpds 1 and 2 were evaluated. Cpd 1 was soluble up to $0.05 \mathrm{mg}$ in $\mathrm{pH} 6.8$ buffer, and human and rat plasma protein binding was $96.9 \%$ and $98.1 \%$, respectively. Pharmacokinetic evaluation of cpd 1 in male Sprague-Dawley rats $(1 \mathrm{mg} / \mathrm{kg}$ i.v.; $3 \mathrm{mg} / \mathrm{kg}$ by oral gavage) yielded an i.v. blood half-life of 2.4 hours, blood clearance of $23 \mathrm{ml} / \mathrm{min} / \mathrm{kg}$, and a volume of distribution of $3.71 / \mathrm{kg}$. The compound was completely orally bioavailable.

In contrast, cpd 2 was not suitable for in vivo application due to high lipophilicity $\left(\log _{\mathrm{p}}>7\right)$, high rat microsomal clearance $(693 \mu 1 / \mathrm{min} / \mathrm{mg})$ and poor solubility $(0.001 \mathrm{mg} / \mathrm{ml} ; \mathrm{pH} 6.8 \mathrm{buffer})$. Based on these data, cpd 1 was used for in vivo profiling.

Pharmacological inhibition of RORC reduces DTH in the rat. DTH was elicited in Lewis rats by an immunization/challenge protocol. Two weeks after intradermal immunization with methylated BSA (mBSA) as antigen in the presence of complete Freund's adjuvant, mBSA was injected into the right ears. Cpd 1 was orally administered at 3 or $10 \mathrm{mg} / \mathrm{kg}$ bis in die (b.i.d.) for 2 days, starting just prior to mBSA challenge. A neutralizing anti-IL-17A antibody was used as comparator, based on reduced DTH responses in Il17-deficient $\left(I l 17^{/-}\right)$mice (22). Blockade of IL-17A by the antibody reduced the DTH ear swelling 
A

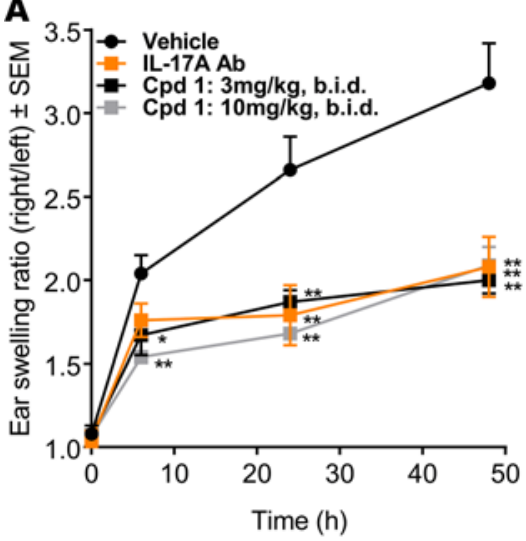

B

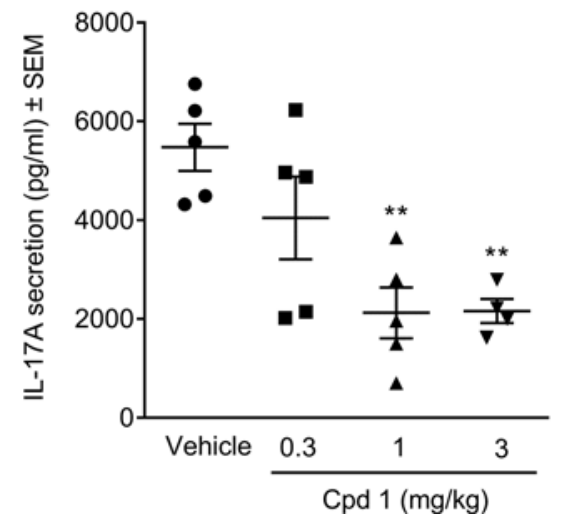

C

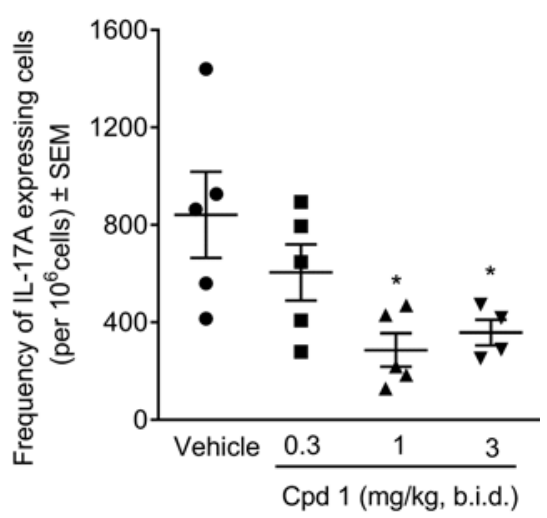

Figure 4. Impaired methylated BSA-induced (mBSA-induced) delayed-type hypersensitivity (DTH) responses in female Lewis rats by the retinoic-acid-orphan-receptor-C (RORC) inhibitor cpd 1. Rats were immunized with mBSA/CFA. Two weeks later, the right ears of the animals were challenged with mBSA, while the left ears were treated with vehicle (5\% glucose). Immediately prior to challenge, bis in die (b.i.d.) dosing of cpd 1 was started at the indicated doses and continued until the end of the studies. (A) Ear swelling was monitored for 48 hours. An IL-17A-specific antibody $(10 \mathrm{mg} / \mathrm{kg}$ s.c. dosed 1 day before mBSA challenge) was used as a reference in another group of rats. The mean \pm SEM of the thickness ratios between mBSA-challenged and vehicle-treated ears are shown $(n=5)$. Cpd 1 blood concentration 2 hours after the last dosing was 334 and 1,286 nM at 3 and $10 \mathrm{mg} / \mathrm{kg}$ b.i.d., respectively. (B and C) Draining lymph node cells were prepared 48 hours after mBSA challenge and stimulated ex vivo with mBSA. (B) IL-17A concentration in supernatants and (C) frequencies of IL-17A-secreting cells were quantified by ELISA and ELISpot after 72 and 24 hours, respectively. Individual data and mean \pm SEM are depicted $(n=4-5)$. Cpd 1 concentrations in the draining lymph nodes 2 hours after the last dosing were 104 , 353, and 1,054 nM at 0.3, 1, and $3 \mathrm{mg} / \mathrm{kg}$ b.i.d., respectively. ${ }^{*} P<0.05$; ${ }^{* *} P<0.01$; Dunnett's test.

response by approximately $50 \%$ compared with that in control rats. RORC inhibition by cpd 1 showed comparable reduction. The effect was already maximal at $3 \mathrm{mg} / \mathrm{kg}$ b.i.d. with no further reduction by increasing the dose to $10 \mathrm{mg} / \mathrm{kg}$ b.i.d. (Figure $4 \mathrm{~A}$ ).

The minimal efficacious dose of cpd 1 was $1 \mathrm{mg} / \mathrm{kg}$ b.i.d., as evidenced by $60 \%$ inhibition of IL-17A secretion and IL-17A-producing cells in an ex vivo mBSA antigen restimulation assay using draining lymph node cells (Figure 4, B and C).

Prolonged inhibition of RORC induces progressive thymic alterations in rats within 13 weeks, including thymic cortical hyperplasia. We conducted a combined 4- and 13-week in vivo preclinical safety study in Wistar Han rats treated with cpd 1 at $10 \mathrm{mg} / \mathrm{kg}$ b.i.d., i.e., at 10-fold minimal efficacious dose. Plasma exposure after 13 weeks was at 21,567 nM.h (AUC) and 6,557 nM (maximum concentration $\left[\mathrm{C}_{\max }\right]$ ). Clinical observations, body weight, food consumption, and clinical pathology data did not show relevant unequivocally compound-related effects, indicating that the treatment was generally well tolerated. While an extensive list of organs was examined, there were consistent histopathological findings only in the thymus after 4 and 13 weeks.

Thymi of rats were histologically characterized by a progressive increase the cortical/medullary ratio (Figure

Table 1. Thymocyte and thymic CD8+ single-positive (SP) cell numbers in rats treated with cpd 1 for 4 and 13 weeks (average \pm SD)

Thymocyte number $\left(1 \times 10^{6}\right)$ Number of CD8 ${ }^{+}$SP cells $\left(1 \times 10^{6}\right)$

$\begin{array}{lcc}\text { Vehicle, } 4 \text { wk } & 306 \pm 96 & 12 \pm 5 \\ \text { Cpd 1, } 4 \text { wk } & 260 \pm 98 & 33 \pm 15^{\mathrm{A}} \\ \text { Vehicle, } 13 \text { wk } & 242 \pm 77 & 9 \pm 3 \\ \text { Cpd 1, 13 wk } & 217+127 & 49 \pm 32^{\mathrm{B}}\end{array}$

Cpd 1 was administered to male Wistar Han rats for 4 and 13 weeks at $10 \mathrm{mg} / \mathrm{kg}$ b.i.d. by oral gavage $(n=9-10)$. At necropsy, thymus samples were taken for determination of thymocyte counts and flow cytometric analysis. Thymocyte counts were calculated as cell number/weight of thymus fragment used for flow cytometry $\times$ total thymus weight. Numbers of $\mathrm{CD} 8^{+} \mathrm{SP}$ cells were calculated as thymocyte number $\times$ frequency of $\mathrm{CD}^{+}{ }^{+} \mathrm{CD} 4^{-}$cells (defined as shown in Figure $6 \mathrm{~A}$ ). ${ }^{\mathrm{A} P}<0.001 ;{ }^{\mathrm{B} P}<0.01 ; t$ test vehicle vs. cpd 1 per time point.
5A) and relative presence of larger thymocytes (Figure 5B) following 4- and 13-week treatment with cpd 1. Strikingly, the thymus of one rat treated for 13 weeks showed cortical lymphoid hyperplasia. In this thymus, normal cortico-medullary demarcation was focally lost, the relative presence of larger thymocytes was particularly prominent, and the population of cells was largely uniform (Figure 5C). Further immunohistochemical characterization of this thymus revealed a clear decrease in the expression of IKAROS (Figure 5D), a marker whose expression is known to be altered in several neoplastic conditions, including $\mathrm{T}$ cell lymphomas (23). Such a decrease of IKAROS was not observed in the thymi of the other cpd 1-treated rats.

Numerical and flow cytometric analysis revealed a slight reduction of the average total thymocyte number and a highly significant and progressive increase in 


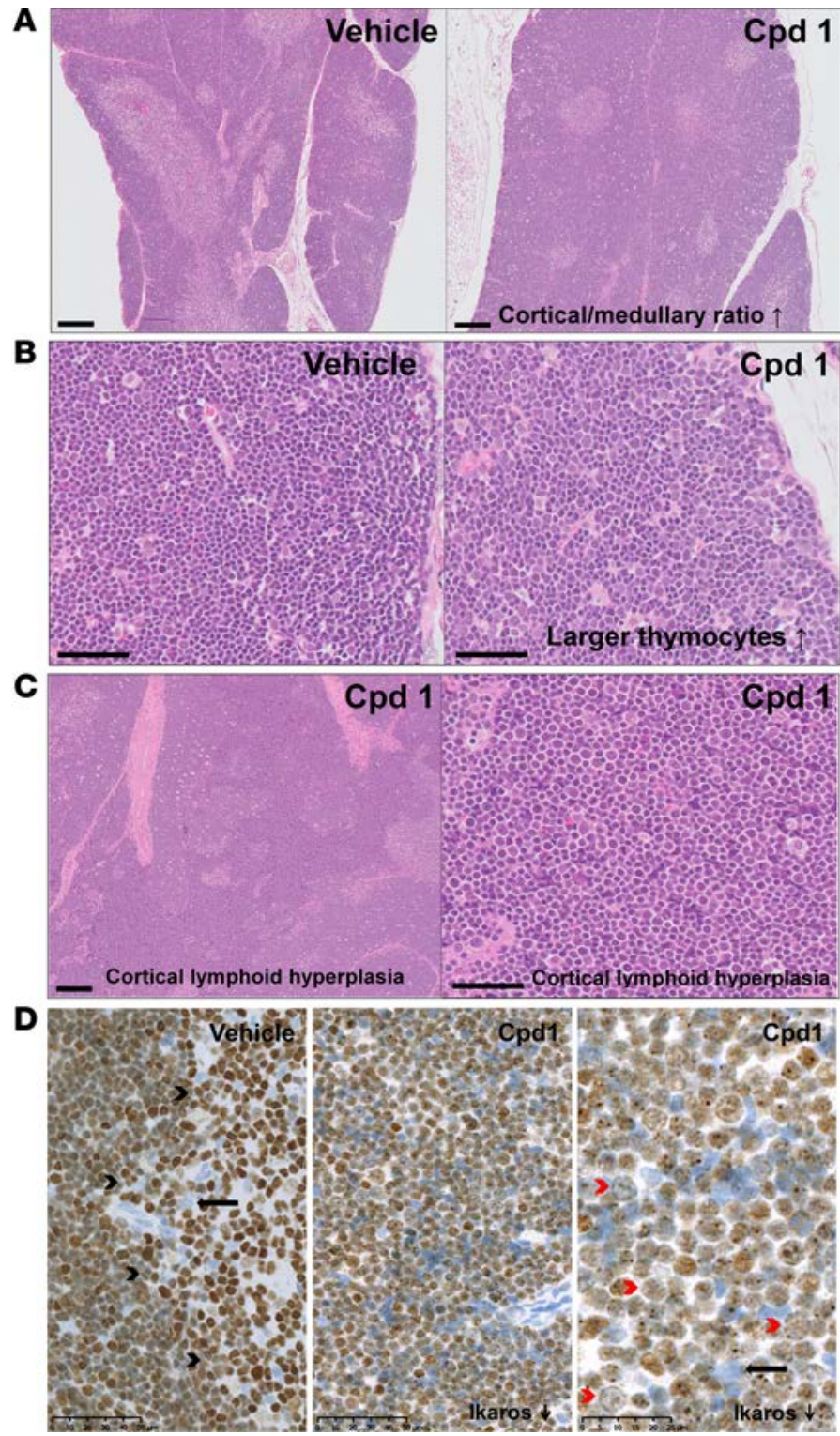

Figure 5. Histopathological analysis of thymic alterations in rats treated with retinoic-acid-orphan-receptor-C (RORC) inhibitor cpd 1 for 13 weeks compared with vehicle control. (A) Subgross view of H\&Estained sections of thymus from vehicle- (left) or cpd 1-treated (right) rats. Cpd 1-induced increase in thymic cortical/medullary ratio (scale bar: $200 \mu \mathrm{m}$ ). (B) Higher magnification showing the cpd 1-induced increase of relative presence of larger thymocytes in cpd 1-treated (right) compared with vehicle-treated (left) rat (scale bar: $40 \mu \mathrm{m}$ ). (C) H\&E-stained section of thymus from cpd 1-treated rat showing cortical lymphoid hyperplasia. Subgross view revealed that the normal cortico-medullary demarcation was focally lost (left, scale bar: $200 \mu \mathrm{m}$ ) and a monomorphic population of large round cells was present (right, scale bar: $50 \mu \mathrm{m}$ ). (D) IKAROS expression in thymus from vehicle- (left) or cpd 1-treated rat showing cortical lymphoid hyperplasia (middle and right). IKAROS expression was reduced in the cortical hyperplastic area. Black arrowheads point to cortical-medullary demarcation, red arrowheads point to lymphoid cells that lost IKAROS, and arrows point to unstained epitheloid cell. $n=9-10$.

numbers of $\mathrm{CD}^{+} \mathrm{SP}$ cells in cpd 1-treated rats after 4 and 13 weeks (Table 1). Similarly to $\operatorname{RorC}^{-1-}$ mice $(11,12)$, also in the case of RORC inhibitor-treated rats, the numbers and frequencies of thymic $\mathrm{CD}^{+} \mathrm{SP}$ cells are difficult to determine because the population of DP cells exhibited reduced levels of CD4 and therefore began to overlap with the $\mathrm{CD}^{+} \mathrm{SP}$ population (Figure $6 \mathrm{~A}$ ). Interestingly, the number of $\mathrm{CD} 8^{+} \mathrm{SP}$ cells was particularly high in the thymus of the rat characterized by thymic cortical hyperplasia (59 million; compare figure [c.f.] Table 1). Furthermore, the frequency of thymocytes in the S/G2/M cell cycle phase was progressively increased in cpd 1-treated rats after 4 and 13 weeks, as determined by flow cytometric analysis of propidium iodide-stained cell suspensions (Figure 6B). Immunohistochemical characterization revealed increased mitosis in thymi of cpd 1-treated rats after 13 weeks, as detected by phosphohistone $\mathrm{H} 3$ (PHH3) serine-10 staining (Figure 6, C and D).

We explored if there was a major increase of a specific $\mathrm{T}$ cell clone by characterizing their $\mathrm{T}$ cell receptor (TCR) profile. We amplified the 20 rat $\mathrm{V} \beta$ genes (V $\beta 1-4, \mathrm{~V} \beta 5.1 / 5.2, \mathrm{~V} \beta 6-7$, $\mathrm{V} \beta 8.1 / 8.2 / 8.5 / 8.6, \mathrm{~V} \beta 9-20)$ by RT-PCR and determined their pattern by spectratyping (poly-, oligo-, or monoclonal). We did not identify any mono- or oligoclonal signal correlating with cpd 1 treatment for 13 weeks in any of the V $\beta$ genes analyzed, whereas a control rat $\mathrm{T}$ cell line showed a clear oligoclonal peak for V $\beta 8.1 / 8.2$ (Supplemental Figure 2).

Interestingly, an increased relative presence of larger thymocytes (accompanied by thymic lymphocytolysis), a higher fraction of $\mathrm{CD}^{+} \mathrm{SP}$ cells, and an increased frequency of thymocytes in the S/G2/M cell cycle phase were already observed after only 1 week of treatment with cpd 1 in a separate pharmacology study in rats at a dose of $30 \mathrm{mg} / \mathrm{kg}$ (data not shown).

RORC inhibitors enhance mouse and cynomolgus thymocyte apoptosis in vitro. Cpd 1 and cpd 2 induced a significant increase in apoptotic mouse DP thymocyte cells (Figure 7A), which was comparable to dexamethasone, a well-known inducer of thymocyte apoptosis (24) used as assay positive control. Induction of apoptosis by inhibition of RORC was confined to immature DP cells, which is the subset where RORC is predominantly expressed, whereas dexamethasone-induced apoptosis was increased in all thymocyte subsets (Table 2). Both compounds accelerated spontaneous apoptosis in a concentration-dependent manner in mouse DP thymocytes after 18 hours of incubation with half maximal effective concentration $\left(\mathrm{EC}_{50}\right.$ ) of 342 and $225 \mathrm{nM}$ for cpd 1 and $\mathrm{cpd}$ 2, respectively. An inactive derivative of cpd 1 did not have a proapoptotic effect, indicating that this process is driven by a RORC-dependent mechanism, also supported by the fact that $\mathrm{RorC}^{-1-}$ thymocytes undergo accelerated spontaneous apoptosis in culture $(11,12,14)$ 
Table 2. Influence of retinoic-acid-orphan-receptor-C (RORC) inhibitors on apoptosis rate among mouse thymocyte subsets in vitro (average \pm SD)

\begin{tabular}{lccc}
\hline & \multicolumn{3}{c}{ Frequencies of Annexin $\mathbf{V}^{+}$cells (\%) } \\
& CD4+ SP & CD8 $^{+}$SP & DP \\
DMSO & $18.7 \pm 4.6$ & $32.4 \pm 6.0$ & $20.2 \pm 5.0$ \\
Dexamethasone & $83.6 \pm 9.8^{\mathrm{A}}$ & $74.4 \pm 3.9^{\mathrm{A}}$ & $87.3 \pm 10.2^{\mathrm{A}}$ \\
$1 \mu \mathrm{M}$ & & & \\
Cpd 1 & $17.5 \pm 1.6$ & $25.2 \pm 1.0$ & $48.1 \pm 0.7^{\mathrm{B}}$ \\
$10 \mu \mathrm{M}$ & & & \\
Cpd 2 & $20.9+4.6$ & $26.3+3.0$ & $49.0 \pm 8.8^{\mathrm{B}}$ \\
$10 \mu \mathrm{M}$ & & &
\end{tabular}

Thymocytes from a C57BL/6 mouse were cultured in triplicates either without addition of compound (DMSO control) or in the presence of dexamethasone or RORC inhibitors (cpd 1, cpd 2) for 18 hours. $\mathrm{CD} 4^{+}$single-positive (SP), $\mathrm{CD} 8^{+} \mathrm{SP}$, and $\mathrm{CD} 4^{+} \mathrm{CD} 8^{+}$doublepositive (DP) subsets were analyzed for Annexin $V$ binding by flow cytometry. ${ }^{A} P<0.0001 ;{ }^{B} P<0.01$; Dunnett's test.
Similarly, cpd 1 induced a time-dependent increase of spontaneous apoptosis in DP thymocytes from a cynomolgus monkey (Figure 7B and Table 3). Because of the limited availability of fresh cynomolgus thymus, we were only able to conduct one kinetic study using cpd 1 at a single concentration

It was previously reported that thymocytes from $\mathrm{Rorc}^{-1-}$ mice almost completely lack expression of the antiapoptotic protein BCL- $\mathrm{X}_{\mathrm{L}}$ whose expression seems to be required for the survival of DP thymocytes and their protection from accelerated apoptosis $(11,12)$. Bcl2l1 mRNA expression was also significantly reduced in thymi of adult inducible Rorc $^{1-}$ mice (16). qPCR analysis of mouse thymocytes revealed that 10 $\mu \mathrm{M}$ of cpd 1 or cpd 2 suppressed Bcl2l1 mRNA expression levels following 3- and 6-hour incubation (Figure 7C). Similar results were obtained in the cynomolgus monkey in vitro system following incubation of thymocytes with cpd 1 (Figure 7D).

The results observed in vitro clearly show that RORC inhibition induced spontaneous apoptosis, as well as downregulation of Bcl2l1 and $B C L 2 L 1$ mRNA expression in rodent and cynomolgus monkey thymocytes, respectively, recapitulating the findings reported for $\mathrm{RorC}^{-1-}$ mice.

\section{Discussion}

RORC is considered as an attractive drug target based on its essential role in the regulation of the Th17 pathway that drives various autoimmune diseases (1-3). Using 2 potent RORC inhibitors from different chemical classes, we show here that they potently block human and rat Th17 differentiation in vitro and attenuate RORC-dependent pro-inflammatory cytokine gene expression levels. Furthermore, for the one compound

Figure 6. Thymocyte changes in rats treated with retinoic-acid-orphan-receptor-C (RORC) inhibitor cpd 1 for $\mathbf{4}$ and $\mathbf{1 3}$ weeks. (A) Inhibition of RORC in rats causes reduced $\mathrm{CD} 4$ expression levels on $\mathrm{CD}_{4}{ }^{+} \mathrm{CD} 8^{+}$double-positive thymocytes resulting in increased frequencies of $\mathrm{CD} 8^{+}$ single-positive thymocytes. Thymocytes prepared from rats treated with vehicle or cpd 1 for 4 and 13 weeks were stained with fluorochrome-labeled antibodies against CD4 and CD8 and analyzed by flow cytometry. Representative cytograms are shown $(n=9-10)$. Average frequencies \pm SD for the cell populations defined by the shown regions are indicated. (B) Inhibition of RORC in rats causes increased frequencies of thymocytes in the S/G2/M cell cycle phases. Thymocytes prepared from rats treated with vehicle or cpd 1 for 4 and 13 weeks were fixed, stained with propidium iodide, and analyzed by flow cytometry. Individual data and mean \pm SD are depicted $(n=9-10)$. (C and $\mathbf{D})$ Inhibition of RORC in rats causes increased thymocyte mitosis, mostly in the cortical areas. Thymus sections from rats treated with vehicle or cpd 1 for 4 and 13 weeks were stained by IHC with an antibody against phosphohistone $\mathrm{H} 3$ ( $\mathrm{PHH} 3$ ) and counterstained with Hematoxylin II and Bluing agent. The result of a digital quantitative analysis is shown in C (unit cells $/ \mathrm{mm}^{2}$; individual data and mean \pm SD are depicted; $n=9-10$ ). Representative stainings of thymus sections from rats treated with vehicle or cpd 1 for 13 weeks are shown in $\mathbf{D}$.
A

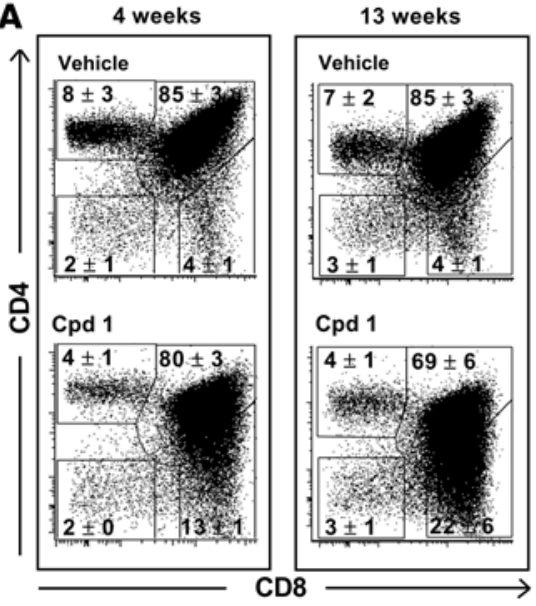

C

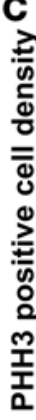

B

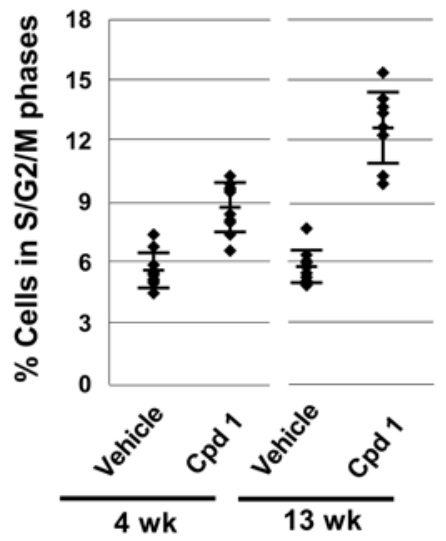

D

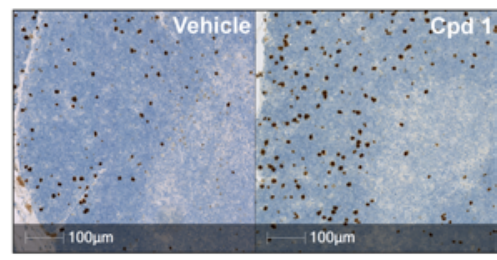


Table 3. Influence of retinoic-acid-orphan-receptor-C (RORC) inhibitors on apoptosis rate among cynomolgus thymocyte subsets in vitro (average \pm SD)

\begin{tabular}{lccc}
\hline & \multicolumn{3}{c}{ Frequencies of Annexin $\mathbf{V}^{+}$cells (\%) } \\
& CD4+ SP & CD8 $^{+}$SP & DP \\
DMSO & $13.0 \pm 1.3$ & $18.6 \pm 0.7$ & $18.0 \pm 1.6$ \\
Dexamethasone & $28.2 \pm 0.3^{\mathrm{A}}$ & $34.6 \pm 0.5^{\mathrm{A}}$ & $57.5 \pm 2.5^{\mathrm{A}}$ \\
$1 \mu \mathrm{M}$ & & & \\
Cpd 1 & $13.7+0.8$ & $18.5+1.3$ & $55.5 \pm 1.6^{\mathrm{A}}$ \\
$10 \mu \mathrm{M}$ & & &
\end{tabular}

Thymocytes from a cynomolgus monkey were cultured in triplicates either without addition of compound (DMSO control) or in the presence of dexamethasone or RORC inhibitor cpd 1 for 18 hours. $\mathrm{CD} 4^{+}$single-positive (SP), $\mathrm{CD} 8^{+} \mathrm{SP}$, and $\mathrm{CD} 4^{+} \mathrm{CD} 8^{+}$doublepositive (DP) subsets were analyzed for Annexin $\mathrm{V}$ binding by flow cytometry. ${ }^{A} P<0.0001$; Dunnett's test. tested in a rat in vivo model (i.e., cpd 1), we demonstrate reduced DTH responses similarly to an anti-IL-17A antibody. This is consistent with previous reports describing RORC inhibitors blocking Th17 differentiation in vitro and beneficial effects of RORC inhibition in rodent in vivo models of experimental autoimmune encephalomyelitis, imiquimod-induced cutaneous inflammation, and collagen-induced arthritis (2-7). The DTH model on cpd 1 allowed us to estimate the minimal efficacious dose, which guided the dose selection for the 4- and 13-week in vivo preclinical safety study in rats.

Following 4 and 13 weeks of b.i.d. administration of the RORC inhibitor cpd 1 to rats, an extensive list of organs was examined histologically. Consistent, unequivocally compound-induced histopathological changes were detectable in the thymus but not in other tissues, including the GI tract, in which a number of RORC-expressing lymphocytes reside. Hence, cpd 1 appears not to generally affect intestinal integrity despite potential changes in IL-17 production in the gut. Recently, it has been shown that pharmacological inhibition of RORC is therapeutically efficacious in a mouse model of intestinal inflammation and selectively reduces cytokine production from Th17 cells, but it preserves innate lymphoid cells in a mouse intestinal infection model (25).

Genetic Rorc deficiency in mice causes strong perturbations of the immune system, as well as development of metastasizing thymic T cell lymphomas $(1,10,11,12,14)$. Development of thymic lymphoblastic lymphoma was also observed in adult inducible $\mathrm{RorC}^{\prime-}$ mice (16). RORC ${ }^{\prime-}$ patients (15) display immunological features similar to RorC $^{-1}$ mice, but so far, the development of thymic $\mathrm{T}$ cell lymphomas has not been described. The thymus in Rorc $^{-1}$ mice shows specific aberrations prior to the development of $\mathrm{T}$ cell lymphomas. Here, we show that pharmacological inhibition of RORC in the rat led to similar, progressive thymic alterations within 13 weeks (c.f. 11, 12, 14 for comparison to orc $^{\text {-1- }}$ mice), including early lymphocytolysis and increasing cortical/medullary ratio with decreased small-size/large-size cell ratio. Importantly, the thymus of one rat treated for 13 weeks was histologically characterized by cortical lymphoid hyperplasia with focal loss of the normal cortico-medullary demarcation, which may represent a preneoplastic change (26). This cortical lymphoid hyperplasia did not represent the common reactive focal hyperplastic nodules seen in association with other lesions (e.g. thymomas) and in the context of thymus atrophy in rats (26). It was rather diffuse with no other changes in the thymus except those induced by the compound. Therefore, the observed hyperplastic lesion most likely lies within a progressive continuum of RORC inhibitor-induced changes, recapitulating those observed in Rorc $^{\prime-}$ mice, and is not a stochastic event. Further similarities to the changes in $\mathrm{RorC}^{-1}$ mice include slightly reduced total thymocyte numbers; progressively increased numbers of $\mathrm{CD}^{+} \mathrm{SP}$ cells (or reduced levels of CD4 on DP cells), particularly strongly in the thymus of the rat characterized by thymic cortical hyperplasia; and progressively increased frequencies of thymocytes in the S/G2/M cell cycle phase. An increased frequency of cells in M phase was confirmed by PHH3 serine-10 IHC in rat thymi following 13-week treatment with cpd 1. In a mouse model of collagen-induced arthritis, the RORC inhibitor SR2211 has also been described to induce reduced total thymocyte numbers and increased frequencies of $\mathrm{CD} 8^{+} \mathrm{SP}$ cells (6). Taken together, the similarities of thymic alterations in RORC inhibitor-treated rodents and genetically $\mathrm{Rorc}^{-1-}$ mice are striking. Moreover, pharmacological inhibition of RORC, similarly to Rorc deficiency in mice, enhanced mouse and cynomolgus monkey DP thymocyte apoptosis, associated with reduced expression of the $\mathrm{mRNA}$ of the anti-apoptotic protein $\mathrm{BCL}-\mathrm{X}_{\mathrm{L}}$, indicating translatability of RORC inhibitor-induced alterations to nonhuman primates.

The high incidence of thymic $\mathrm{T}$ cell lymphomas in $\mathrm{RorC}^{-1-}$ mice may be a result of dysfunction of important physiological events during thymocyte maturation. Enhanced apoptosis of DP cells (facilitated by suppressed expression of BCL-X $\mathrm{L}_{\mathrm{L}}$, as well as decreased expression of $\mathrm{p} 27^{\mathrm{kip} 1}$ and correspondingly increased activity of the cyclin-dependent kinase 2 (CDK2), appear to play important roles, favoring the expansion of potentially immature $\mathrm{CD}^{+} \mathrm{SP}$ cells in the thymic cortex $(11,12,14)$. Interestingly, cell competition has been described as a tumor suppressor mechanism in the thymus (27), and a related mechanism may also contribute to the development of $\mathrm{T}$ cell lymphomas in $\mathrm{RorC}^{-1-}$ mice. Clonality was not observed in thymi of RORC inhibitor-treated rats within 13 weeks, not even in the case of the rat characterized by thymic cortical hyperplasia, but this may become apparent only at a later stage. However, immunohistochem- 

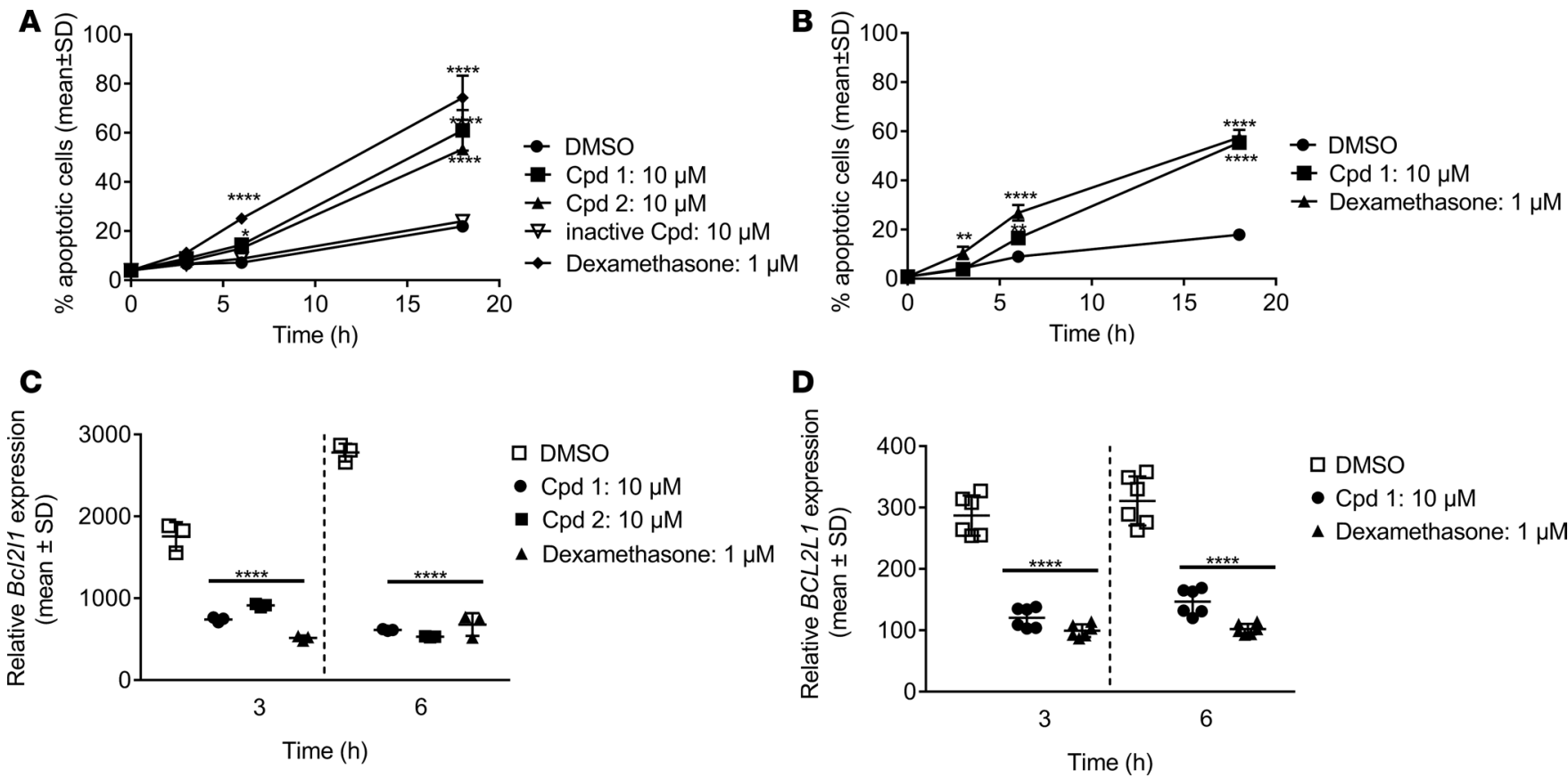

Figure 7. Inhibition of retinoic-acid-orphan-receptor-C (RORC) accelerates spontaneous apoptosis in mouse and cynomolgus monkey thymocytes in vitro. Freshly isolated thymocytes from $\mathbf{C 5 7 B L / 6}$ mice (A and $\mathbf{C}$ ) or cynomolgus monkey (B and $\mathbf{D}$ ) were cultured either without addition of compound or in the presence of RORC inhibitors or dexamethasone for up to 18 hours. (A and B) At different time intervals, cells were analyzed for Annexin $V$ binding by flow cytometry. The percentage of $C D 4^{+} C D 8^{+}$double-positive thymocytes that underwent apoptosis induced by the RORC inhibitors was calculated and plotted. (C and $\mathbf{D})$ Following 3 and 6 hours incubation time, cells were analyzed for (C) Bc/2/1 or (D) BCL2L1 mRNA expression by qPCR. Representative examples of (C) triplicate readings from 3 mice or (D) triplicate readings of assay duplicates from 1 cynomolgus monkey are shown. Individual data and means \pm SD are depicted. ${ }^{*} P<0.05$; ${ }^{* *} P<0.01 ;{ }^{* * *} P<0.0001$; Dunnett's test.

ical staining revealed increased mitosis and a decreased expression (or, in individual cells, even a complete loss) of IKAROS in the thymus of the rat with thymic cortical hyperplasia. These characteristics suggest neoplastic transformation. IKAROS (also known as Lyf-1) is a lymphoid-specific transcription factor, not only important for normal hematopoiesis, but also implicated as tumor suppressor. Rapid development of $\mathrm{T}$ cell lymphoma was detected in mice with aberration in Ikaros expression (28), and Ikaros deletions/mutations are frequently seen as directly involved in development of thymic lymphoma (23).

In adult humans, the thymus shrinks at a rate of approximately $3 \%$ per year, slowing down with age, resulting in reduced thymic output (29). This process is considered to represent a continuous adaptation to protect the thymus from the development of tumors due to age-dependently reduced influx of lymphoid progenitor cells into the thymus and consequently longer residence time of T cells in the thymic cortex (30). Hence, it cannot be excluded that ,in the case of ongoing age-related thymus involution, RORC inhibitors induce similar thymic aberrations as described above.

In summary, while RORC inhibitors block the development of Th17 cells and their cytokine production across species in vitro and in rats in vivo, they also recapitulate thymic aberrations similar to those observed in $\mathrm{Rorc}^{-1}$ mice prior to the development of thymic T cell lymphomas, even reaching cortical hyperplasia with preneoplastic features in one rat. Chronic studies in rodents and nonrodents, including aged animals with involuted thymi, are warranted to assess whether thymic T cell lymphomas will develop after prolonged therapy with RORC inhibitors to better define the translational risk for humans.

\section{Methods}

Control items. Dexamethasone was obtained from Sigma-Aldrich. The mouse-specific anti-IL-17A antibody BZN035 (31) was generated at Novartis.

Physicochemical properties. Microsomal intrinsic clearance was determined by the rate of drug depletion after incubation at $1 \mu \mathrm{M}$ in male Sprague-Dawley rat liver microsomes (32), plasma protein binding was determined using rapid equilibrium dialysis (33), and solubility was measured according to previously described protocols (34). 
TR-FRET assay. The LBD of human RORC comprising amino acids 264-518 with an N-terminal His6-S-tag was produced at Novartis, the biotinylated RIP140 coactivator peptide (biotinyl-NH-AhxNSHQKVTLLQLLLGHKNEEN-CONH2) was purchased from Thermo Scientific, Europium-labeled anti-6xHis-tag antibody was obtained from Perkin Elmer, and Cy5-streptavidin was purchased from GE Healthcare. Compound dilutions were prepared from $10 \mathrm{mM}$ stock solutions in DMSO (1\% final assay concentration) and were incubated in the TR-FRET assay mixture containing $5 \mathrm{nM}$ recombinant Histagged RORC-LBD, $90 \mathrm{nM}$ biotinylated RIP140 coactivator peptide, $0.45 \mathrm{nM}$ Cy5-labeled streptavidin, and $1.5 \mathrm{nM}$ Europium-labeled anti-6xHis-tag antibody. After incubation of assay plates for 1 hour at room temperature, the TR-FRET donor $(615 \mathrm{~nm})$ and acceptor $(665 \mathrm{~nm})$ emission values were recorded using an Envision 2102 multilabel reader (Perkin Elmer). Concentration-response curves were derived from the $665 / 615 \mathrm{~nm}$ emission ratio, and $\mathrm{IC}_{50}$ values were calculated from concentration-response curves.

$R O R C$-hinge- $L B D$ reporter gene assay. A Jurkat cell line (purchased from the American Type Tissue Collection) stably expressing a pGL4.35 reporter plasmid (Promega) was generated; this plasmid contains 9 repeats of the GAL4 upstream activator sequence, driving the transcription of the luciferase gene. The plasmid containing the human RORC-hinge-LBD (provided by Shin-Shay Tian, The Genomics Institute of the Novartis Research Foundation, La Jolla, California, USA) was cloned into the pFN26A (BIND) hRluc-neo Flexi Vector expressing a fusion protein composed of a DNA-binding domain (DBD) of the $G A L 4$ gene (Promega) to form the GAL4-DBD-RORC-hinge- $L B D$ fusion construct. This vector was transfected into Jurkat cells containing the reporter plasmid to generate clones stably expressing the 2 constructs. To assess selectivity against other ROR family members, fusion plasmid constructs containing human RORA or RORB-LBD and GAL4-DBD (provided by Shin-Shay Tian) were transiently transfected into Jurkat cells $\left(1 \mu \mathrm{g} / 10^{6}\right.$ cells) stably expressing the luciferase reporter plasmid. After 24 hours, cells were prepared for compound testing. Cells were resuspended in RPMI1640 medium containing Glutamax, 25 mM HEPES, $10 \% \mathrm{FCS}, 1 \%$ nonessential amino acids (NEAA), $1 \mathrm{mM}$ sodium pyruvate, $100 \mathrm{U} / \mathrm{ml}$ penicillin, and 100 $\mathrm{mg} / \mathrm{ml}$ streptomycin (Gibco), and were seeded in 96-well plates at $5 \times 10^{4}$ cells/well. The RORC inhibitors or DMSO as a control were added to the cells; after 24 hours of incubation, cells were lysed (Steady lite plus, Perkin Elmer) and luciferase activity was measured using an EnVision Multilabel Plate Reader (Perkin Elmer). The $\mathrm{IC}_{50}$ values for inhibitors were determined using sigmoidal dose-response curve fitting.

Animals. Female C57BL/6 mice (approximately 8-11 weeks old), male Sprague-Dawley rats (approximately 300-350 g at start of dosing), female Lewis rats (approximately 120-150 g at start of dosing), and male Wistar Han rats (approximately 200-350 g at start of dosing) were purchased from Charles River Laboratories. Cynomolgus thymus tissue was obtained from one 4.5-year-old male cynomolgus monkey, purchased from World Wide Primates. Animals were housed in enriched environments with an approximate 12-hour light/dark cycle, and food and water were provided ad libitum.

Human peripheral blood T cell cultures and cytokine analysis. Buffy coats from healthy volunteers were provided under informed consent and collected through the InterRegionale Blutspende of the Swiss Red Cross. Peripheral blood mononuclear cells (PBMCs) were isolated by density gradient centrifugation using FicollPaque. After washing, adherent cells were removed by incubation of cells in plastic culture flasks for 30 minutes at $37^{\circ} \mathrm{C}$. $\mathrm{CD} 4^{+}$or $\mathrm{CD} 8^{+} \mathrm{T}$ cells were obtained by immunomagnetic isolation using a $\mathrm{CD} 4^{+}$or $\mathrm{CD} 8^{+}$ $\mathrm{T}$ cell Enrichment Kit according to the manufacturer's instructions (Stemcell Technologies). Purity of the T cell subsets was assessed by flow cytometry and was routinely $>93 \%$. For polarization experiments, $\mathrm{T}$ cell subsets were seeded at $5 \times 10^{4}$ cells/well in flat-bottom 96-well plates that had been coated with monoclonal antibodies against CD3 (clone OKT-3; BioXcell) and CD28 (clone CD28.2; BioLegend). For Th17 or Tc17 polarization assays, cells were cultured with a cytokine cocktail containing IL-6 (20 ng/ml; catalog 570804; BioLegend), TGF- $\beta 1$ (5 ng/ml; catalog 580704; BioLegend), IL-1 $\beta$ (10 ng/ml; catalog 201-LB-025/ CF; R\&D Systems), and IL-23 (10 ng/ml; catalog 574102; BioLegend). For generation of Th17 cells, naive $\mathrm{CD}^{+} \mathrm{CD} 45 \mathrm{RA}^{+} \mathrm{CD} 45 \mathrm{R}^{-} \mathrm{T}$ cells were obtained by negative immunomagnetic isolation and were stimulated for 7 days with plate-bound CD3/CD28 antibodies and with the Th17 cytokine cocktail. For Th1 polarization assays, cells were incubated with IL-12 (10 ng/ml; catalog 219-IL-005/CF; R\&D Systems) and antiIL-4 antibody ( $5 \mu \mathrm{g} / \mathrm{ml}$; clone MP4-25-D2; BioLegend). For Th2 polarizations, cells were cultured with IL-4 (10 ng/ml; catalog 204-IL-010/CF; R\&D Systems) and anti-IFN antibody (5 g/ml; clone B27; BioLegend). No cytokine cocktail was added for Th0 polarization assays. Cells were grown in IMDM+Glutamax medium (Gibco), supplemented with $10 \%$ FCS, 1\% NEAA, $1 \mathrm{mM}$ sodium pyruvate, $100 \mathrm{U} / \mathrm{ml}$ penicillin, and $100 \mathrm{mg} / \mathrm{ml}$ streptomycin (Gibco). Cell cultures were incubated in the presence of RORC inhibitors or 
DMSO control, and supernatants were collected after 48 or 72 hours of incubation. IL-17A (eBioscience), IL-2 (BioLegend), IL-13 (eBioscience), or IFN- $\gamma$ (BioLegend) cytokine concentrations were quantified by ELISA according to the manufacturer's instructions. The $\mathrm{IC}_{50}$ values for inhibitors were determined by sigmoidal dose-response curve fitting. In the case of additional flow cytometric determination of intracellular cytokines, polarized cells were stimulated for 4 or 8 hours at $37^{\circ} \mathrm{C}$ with $10 \mathrm{ng} / \mathrm{ml}$ PMA (Sigma-Aldrich) and $1 \mu \mathrm{g} / \mathrm{ml}$ ionomycin (Calbiochem) in the presence of RORC inhibitors or DMSO control and $10 \mu \mathrm{g} / \mathrm{ml}$ Brefeldin A (Sigma-Aldrich).

Human $\gamma \delta$ T cell culture. Human $\gamma \delta$ T cells were obtained from PBMCs by immunomagnetic isolation using a human $\gamma \delta \mathrm{T}$ cell isolation kit (Stemcell Technologies) according to the manufacturer's instructions. Purity was assessed by flow cytometry and was $94 \%$. Cells were seeded at $2 \times 10^{5}$ cells/well in flat-bottom 96-well plates and stimulated with PMA $(5 \mathrm{ng} / \mathrm{ml})$ and ionomycin $(0.5 \mu \mathrm{g} / \mathrm{ml})$ in the presence of RORC inhibitors or DMSO control. After 24-hour incubation, samples were prepared for gene expression analysis by RT-PCR.

Isolation of rat $C D 4^{+} T$ cells and Th17 cell polarization assay. $\mathrm{CD}^{+} \mathrm{T}$ cells from male Lewis rats were obtained from splenocytes by incubation of cells with a cocktail of the following rat-specific antibodies: CD8a (clone OX8; BioLegend), CD45RA (clone OX33; BioLegend), CD11b/c (clone OX42; BD Biosciences), CD25 (clone OX39; eBioscience), and erythrocytes (clone OX83; Cedarlane). After a washing step, the cells were incubated for 15 minutes at $4^{\circ} \mathrm{C}$ with anti-IgG-coated microbeads (Miltenyi Biotec), and unwanted cells were magnetically depleted using a MACS column system according to the manufacturer's instructions (Miltenyi Biotec). The purity of CD4 ${ }^{+} \mathrm{T}$ cells was typically $90 \%-95 \%$ as assessed by flow cytometry. For Th17 cell differentiation assays, CD4+ cells were seeded at $5 \times 10^{4}$ cells/well in 96-well flat-bottom plates and stimulated with precoated rat-specific anti-CD3 (4 $\mathrm{g} / \mathrm{ml}$; clone G4.18; eBioscience) and soluble anti-CD28 antibody ( $2 \mu \mathrm{g} / \mathrm{ml}$; clone JJ319; BioXcell). Cells were cultured under Th17-polarizing conditions using recombinant human TGF- $\beta 1$ (5 ng/ml; catalog 580704; BioLegend), mouse IL-6 (20 ng/ml; catalog 554582; BD Pharmingen), rat IL-1 $\beta$ (10 ng/ml; catalog 501-RL-010/CF; R\&D Systems), and mouse IL-23 (10 ng/ml; catalog 589002; BioLegend) in the presence of anti-rat IFN- $\gamma$ antibody ( $5 \mu \mathrm{g} / \mathrm{ml}$; clone DB-1; BioLegend). After 48 hours of incubation, the supernatants were collected to quantify IL-17A cytokine levels by ELISA according to the manufacturer's instructions (eBioscience). The $\mathrm{IC}_{50}$ values for inhibitors were determined by sigmoidal dose-response curve fitting.

Pharmacokinetics/toxicokinetics/bioanalytics. To quantify cpd 1 and 2, $30 \mu \mathrm{lof}$ plasma or tissue homogenate was mixed with $200 \mu \mathrm{l}$ acetonitrile and centrifuged at $4^{\circ} \mathrm{C}$. Supernatant $(200 \mu \mathrm{l})$ was diluted with 150 $\mu 1$ water. An aliquot was injected into the liquid chromatography-mass spectrometry system for analysis. Data analysis was performed with WinNonlin Phoenix 64 using a noncompartmental approach.

DTH response in rat. Female Lewis rats were sensitized intradermally on the back at 2 sites with $100 \mu \mathrm{g}$ of mBSA (Sigma-Aldrich) homogenized 1:1 with complete Freund's adjuvant (DIFCO). Fourteen days after sensitization, rats were challenged with $10 \mu \mathrm{lof} \mathrm{mBSA}(10 \mathrm{mg} / \mathrm{ml}$ in $5 \%$ glucose) into the right ear and with $10 \mu \mathrm{l}$ of the vehicle ( $5 \%$ glucose) into the left ear. The rat cross-reactive anti-IL-17 antibody BZN035 was administered s.c. at $30 \mathrm{mg} / \mathrm{kg}$ on the day before the mBSA challenge. Cpd 1 was dosed twice daily per oral gavage starting just before the antigen challenge until the end of the study. Ear swelling was measured using digital calipers (Mitutoyo) and expressed as a ratio of right (mBSA-injected) versus left (control) ear thickness. Cells were harvested from the draining auricular lymph nodes 48 hours after challenge and used in ex vivo $\mathrm{T}$ cell recall assays.

Ex vivo $T$ cell recall assays. For determination of IL-17 release to cell culture supernatants, lymph node single-cell suspensions from the DTH studies were seeded into 96-well microtiter plates $\left(4 \mathrm{x} 10^{5}\right.$ cells/well), and antigen-specific T cells were restimulated with mBSA $(200 \mu \mathrm{g} / \mathrm{ml})$ and incubated for 72 hours. Supernatants were collected, and IL-17A concentrations were determined by ELISA according to the manufacturer's instructions (eBioscience). For determination of the frequencies of IL-17A secreting cells, lymph node single-cell suspensions from the DTH studies were seeded at $1 \times 10^{5}$ cells/ well into 96-well PVDF plates (Millipore), precoated with anti-mouse IL-17 capture antibody that is cross-reactive with rat IL-17A (ELISpot development module from product no. SEL421, R\&D Systems). Cells were stimulated with mBSA $(200 \mu \mathrm{g} / \mathrm{ml})$ at $37^{\circ} \mathrm{C}$ for 24 hours. After washing, the plates were incubated with biotinylated anti-mouse IL-17 detection antibody (ELISpot development module from SEL421, R\&D Systems) overnight at $4^{\circ} \mathrm{C}$, followed by incubation with streptavidin-alkaline phosphatase concentrate. The spots were developed with an ELISpot blue color module using BCIP/NBT 
chromogen as the substrate (R\&D Systems). The PVDF membrane plates were dried, and the spots were counted with an ELISpot reader (AID). Quadruplicate wells were averaged and results presented as number of spots $/ 1 \times 10^{6}$ cells.

Four-/13-week repeat-dose rat study. Cpd 1 was administered as a $1 \mathrm{mg} / \mathrm{ml}$ solution in $0.1 \mathrm{M}$ citrate buffer $\mathrm{pH} 2$ to male Wistar Han rats for 4 and 13 weeks at $10 \mathrm{mg} / \mathrm{kg}$ b.i.d. (7- and 17-hour rhythm) by oral gavage at a dose volume of $10 \mathrm{ml} / \mathrm{kg}$. Rats of corresponding control groups were similarly treated with vehicle (100 mM citrate buffer). Blood concentrations of cpd 1 were determined toward the end of the treatment periods at $0.25,0.5,1,3$, and 7 hours after dose ( 2 animals per group at each time point; maximally 3 blood samples per rat). At necropsy, organs/tissues were sampled for histological examination. In addition, thymus samples were taken for determination of organ weights and thymocyte counts (calculated as cell number/mg thymus fragment used for flow cytometry $\times$ total thymus weight), flow cytometric analysis, and $\mathrm{T}$ cell clonality assessment by spectratyping.

In vitro thymocyte apoptosis assays. Single-cell suspensions of thymocytes from female C57BL/6 mice and a male cynomolgus monkey were prepared by passing thymi through $70-\mu \mathrm{m}$ cell strainers (Falcon). The cells $\left(1 \times 10^{6}\right.$ cells/well) were cultured for various times in RPMI1640 medium containing 10\% FCS in the presence or absence of test compounds added from stock solutions in DMSO. The final DMSO concentration in the cell cultured did not exceed $0.1 \%$. All incubations were performed in triplicate. Apoptosis was quantified by flow cytometric analysis. Bcl2l1 or BCL2L1 mRNA expression was determined by qPCR.

Histopathology. Adrenal glands, aorta, brain, esophagus, femur and knee joints, Harderian glands, heart, kidneys, larynx, liver, lungs, mammary gland, mandibular and mesenteric lymph nodes, pancreas, peripheral nerve (sciatic), pituitary, prostate, salivary glands, seminal vesicles, skeletal muscle, skin, small and large intestine, spinal cord, spleen, sternum with bone marrow, stomach, thymus, thyroids with parathyroids, tongue, trachea, ureters, and urinary bladder for each animal were collected and fixed in neutral phosphate buffered formalin up to 8 weeks, with the exception of the thymi and colon, which were fixed for only $72 \pm 48$ hours; epididymides, eyes with optic nerve, and testes were fixed in Modified Davidson's Fixative. All organs were embedded in paraffin wax, sectioned at 4- $\mu \mathrm{m}$ sections, deparaffinized in xylene, and rehydrated through graded ethanol series, followed by staining with H\&E. Immunohistochemical stainings were performed using a rabbit anti-PHH3 antibody (catalog 9701; Cell Signaling Technology) as a cell cycle marker and a rabbit anti-pan-Ikaros antibody (catalog 5443; Cell Signaling Technology) as a tumor suppressor marker. The stainings were performed on Ventana Discovery XT (Roche Diagnostics), and all chemicals were provided by Roche Diagnostics. Briefly, 3- $\mu \mathrm{m}$ paraffin tissue sections from rat thymi were dewaxed, rehydrated, and then subjected to heat-induced antigen retrieval treatment using standard CC1 (Tris-based) for PHH3 and standard antigen retrieval CC2 (citrate-based) for Ikaros. Tissue sections were then incubated for 6 hours with the respective primary antibody. Afterward, the sections were washed and incubated with a horseradish-conjugated (HRP-conjugated) secondary antibody (UltraMap anti-rabbit HRP). Detection was performed using ChromoMap DAB kit according to the manufacturer's recommendations. Counterstaining with Hematoxylin II and Bluing agent was performed. Sections were dehydrated and covered using Eukitt (Medite). The slides were scanned for subsequent image analysis using a Hamamatsu slide scanner (NanoZoomer 2.0 HT, scanning software NDP-Scan version 2.5, Hamamatsu Photonics). Automated quantitative assessment of stain-positive nuclei and negative tissue areas for the 2 stains described above was performed on whole slide scan data using HALO image analysis software (version 2.0, Indica Labs). Thymus tissue has been manually outlined on each digital slide scan. Image pixel classifiers have been used to automatically delineate the cortex as regions for subsequent analysis. For each of the 2 stains, a classifier has been trained in a machine learning process on a subset of the randomly selected digital slides, including scans from all groups, and assigned as training data. For the detection of stain-positive and stain-negative cells, the algorithm CytoNuclear v1.4 has been configured individually for each staining and combined with the respective classifier. As a read-out, the number of stain-positive nuclei divided by the cortex area has been computed from the classification and the cell and area detection.

Flow cytometry. Polarized $\mathrm{T}$ cell subsets from the human peripheral blood $\mathrm{T}$ cell cultures were surface stained with FITC-conjugated anti-CD4 mAb (clone RPA-T4), followed by fixation and permeabilization with Cytofix/Cytoperm (BD Biosciences) and intracellular staining with APC-conjugated anti-IL-2 (clone MQ1-17H12), APC-conjugated anti-IFN- $\gamma$ (clone B27), PE-conjugated anti-IL-4 (clone 8D4-8), or AlexaFluor 647-conjugated anti-IL-17A (clone N49-653) mAb (all antibodies from BD Biosciences). Thymocyte suspensions from the 4/13-week repeat-dose rat study were either incubated with a combination 
of APC-conjugated anti-rat-CD4 (clone OX35; BD Biosciences) and PerCP-conjugated anti-rat-CD8a (clone OX8; BD Biosciences) mAbs for determination and quantification of double-negative (DN), CD4 ${ }^{+}$ SP, $\mathrm{CD}^{+} \mathrm{SP}$, and DP subsets or fixed with $70 \%$ ethanol and stained with propidium iodide (Invitrogen) for cell cycle assessment. Thymocytes from the in vitro apoptosis assays were incubated with anti-CD4 (mouse: clone RM4-5; Alexa488-conjugated; BD Biosciences; cynomolgus: clone OKT4; PE-conjugated; BioLegend) and anti-CD8 (mouse: clone 53-6.7; PE-conjugated; BD Biosciences; cynomolgus: clone SK1; FITC-conjugated; BioLegend) mAbs, followed by washing with Annexin V binding buffer and staining with APC-conjugated Annexin V (mouse: product no. 550474; BD Biosciences; cynomolgus: product no. 640920; BioLegend) and 7-aminoactinomycin D (BioLegend). Flow cytometric analysis of labeled cells was performed with BD Biosceinces flow cytometers, either LSR II, FACSCanto, or LSR Fortessa. Flow cytometry data were analyzed using FACSDiva or FlowJo software.

$T$ cell clonality assessment by spectratyping. T cell clonality was assessed by comparing the spectratypes of thymus samples from vehicle- and cpd 1-treated rats in the 13-week repeat-dose study. Total cellular RNA from a rat $\mathrm{T}$ cell line (Nb2-11, Sigma-Aldrich, catalog 97041101) and from the rat thymi was extracted using RNeasy Mini Kit (Qiagen, catalog 74106) according to manufacturer's instructions. qPCR was performed with $4 \mu \mathrm{g}$ of RNA on a Bio-Rad thermocycler using one-step (Platinum ThermoScript One-Step system, Invitrogen, catalog 11731015) or 2-steps RT-PCR:cDNA conversion with High Capacity kit (Applied Biosystems, catalog 4368813) and PCR with AmpliTaq Gold DNA Polymerase (Applied Biosystems, catalog N8080245). Amplification of rat Eif1a was performed with a commercial Taqman GeneAssay (Rn01491636_m1, Applied Biosystems) and of TCR $\beta$ CDR3 regions with forward V-primers for V $\beta 1-4, \mathrm{~V} \beta 5.1 / 5.2$, V $\beta 6-7, \mathrm{~V} \beta 8.1 / 8.2 / 8.5 / 8.6$, V $\beta 9-20$ (35), and reverse C-primer (36) synthesized at Microsynth AG using FAM as fluorescent dye. PCR product sizes were measured in an Agilent ScreenTape System (D1000 kit; catalogs 5067-5582 and 5067-5583) or a Shimadzu MultiNA (DNA1000 kit; catalogs 292-27911-28 and 292-36010-49) following the manufacturers' instructions. Spectratypes were determined using FAM-labeled reverse primer in 3730xl DNA Analyzer (Applied Biosystems) using Hi-Di Formamide (Applied Biosystems, catalog 4311320) and Genescan-1200 LIZ dye Size Standard (Applied Biosystems, catalog 4379950) following supplier's protocol. Results were visualized using Genemapper Software version 5 (Applied Biosystems).

qPCR for Bcl2l1 and BCL2L1 mRNA, as well as Th17 signature gene expression. Total RNA from mouse or cynomolgus monkey thymocytes, or from human Th17 or $\gamma \delta \mathrm{T}$ cells, was extracted using an RNeasy kit, including a DNAse I digestion step according to the manufacturer's instructions (Qiagen). cDNAs were prepared using the High Capacity cDNA Reverse Transcription Kit (Applied Biosystems). qPCR analysis was performed using a TaqMan ViiA7 (Applied Biosystems). The level of each gene was normalized to $18 S$ rRNA (4319413E) or Gus expression (431088E) using the $\Delta \Delta \mathrm{Ct}$ method. The following probes were used for RT-PCR. Macaca fascicularis: RORC (Mf01076115_m1), BCL2L1 (Mf02621774_s1). Mus musculus: Rorc (Mm00441139_m1), Bcl2l1 (Mm00437783_m1). Homo sapiens: RORC (Hs01076112_m1), IL17A (Hs00936345_m1), IL17F (Hs00369400_m1), IL22 (Hs01574154_m1), IL26 (Hs00218189_m1), IL23R (Hs00332759_m1), CCR6 (Hs00218189_m1).

Statistics. The means were compared using both ANOVA followed by Dunnett's test for multiple comparisons and unpaired 2-tailed $t$ test for comparison of 2 groups (GraphPad Software Corporation Inc.). $P<0.05$ was considered significant.

Study approval. Animal housing and studies were performed in accordance with the Swiss Animal Welfare law, with the EU Animal Health regulations, in particular Council Directive no. 2010/63/EU of 22-Sep-2010 on the protection of animals used for scientific purposes, or with the United States Department of Agriculture Animal Welfare Regulations, ensuring humane treatment and care of laboratory animals.

\section{Author contributions}

Research studies were designed and data were analyzed and interpreted by CG, AP, MLH, DT, TRS, ADRE, LD, AB, KK, SH, JD, RS, DDP, AD, MD, and JS. KH and DO were responsible for compound design and synthesis. CG and JS wrote the manuscript with contributions from all other authors.

\section{Acknowledgments}

We thank the Novartis colleagues Elodie Arduin, Alex Bussenault, Brigitte Christen, Katy Darribat, Valérie Dubost, Florence Ecoeur, Yves Henriquez, Cornelia Heuberger, Jeremy Houguenade, Bernhard Jost, 
Raphaela Kutil, Ina Mastalurova, Jill Omerza, Julia C. Riker, Lukas Roth, René Schaffner, Jeannine Streich, Anja Walkam, and Jessica Weiss for technical assistance. We thank the scientific and technical staff of CiToxLab for their collaboration and support, especially Josée Perron. We thank the Novartis colleagues Dominique Brees, Karin Briner, Christian Bruns, Nigel G. Cooke, Peter Heining, Rene Hersperger, Michael Kammüller, Daniel Stiehl, Elaine Tritto, and Juergen Wagner for helpful advice and scientific support. All studies were internally paid by Novartis.

Address correspondence to: Jens Schümann, Preclinical Safety, Novartis Institutes for BioMedical Research, 4002 Basel, Switzerland. Phone: 41.61.3243628; E-mail: jens.schuemann@novartis.com.

1. Jetten AM. Retinoid-related orphan receptors (RORs): critical roles in development, immunity, circadian rhythm, and cellular metabolism. Nucl Recept Signal. 2009;7:e003.

2. Huh JR, Littman DR. Small molecule inhibitors of ROR $\gamma t$ t targeting Th17 cells and other applications. Eur J Immunol. 2012;42(9):2232-2237.

3. Kojetin DJ, Burris TP. REV-ERB and ROR nuclear receptors as drug targets. Nat Rev Drug Discov. 2014;13(3):197-216.

4. Xiao S, et al. Small-molecule ROR $\gamma$ t antagonists inhibit T helper 17 cell transcriptional network by divergent mechanisms. Immunity. 2014;40(4):477-489.

5. Skepner J, et al. Pharmacologic inhibition of ROR $\gamma$ t regulates Th17 signature gene expression and suppresses cutaneous inflammation in vivo. J Immunol. 2014;192(6):2564-2575.

6. Chang MR, Lyda B, Kamenecka TM, Griffin PR. Pharmacological repression of ROR $\gamma$ is therapeutic in the collagen-induced arthritis experimental model. Arthritis Rheumatol. 2014;66(3):579-588.

7. Wang Y, et al. Discovery of novel N-(5-(arylcarbonyl)thiazol-2-yl)amides and N-(5-(arylcarbonyl)thiophen-2-yl)amides as potent ROR $\gamma$ t inhibitors. Bioorg Med Chem. 2014;22(2):692-702.

8. An Ascending Multiple Dose Study With VTP-43742 in Healthy Volunteers and Psoriatic Patients. ClinicalTrials.gov. https://clinicaltrials.gov/ct2/show/NCT02555709?term=VTP-43742\&rank=1. Accessed February 8, 2017.

9. Study of Safety and Efficacy of Topical GSK2981278 Ointment in Plaque Psoriasis. ClinicalTrials.gov. https://clinicaltrials. gov/ct2/show/NCT02548052?term=GSK2981278\&rank=1. Accessed February 8, 2017.

10. Egawa T, et al. Genetic evidence supporting selection of the Valpha14i NKT cell lineage from double-positive thymocyte precursors. Immunity. 2005;22(6):705-716.

11. Sun Z, et al. Requirement for RORgamma in thymocyte survival and lymphoid organ development. Science. 2000;288(5475):2369-2373.

12. Kurebayashi S, et al. Retinoid-related orphan receptor gamma (RORgamma) is essential for lymphoid organogenesis and controls apoptosis during thymopoiesis. Proc Natl Acad Sci USA. 2000;97(18):10132-10137.

13. Guo J, et al. Regulation of the TCRalpha repertoire by the survival window of CD4(+)CD8(+) thymocytes. Nat Immunol. 2002;3(5):469-476.

14. Ueda E, Kurebayashi S, Sakaue M, Backlund M, Koller B, Jetten AM. High incidence of T-cell lymphomas in mice deficient in the retinoid-related orphan receptor RORgamma. Cancer Res. 2002;62(3):901-909.

15. Okada S, et al. IMMUNODEFICIENCIES. Impairment of immunity to Candida and Mycobacterium in humans with bi-allelic RORC mutations. Science. 2015;349(6248):606-613.

16. Liljevald $\mathrm{M}$, et al. Retinoid-related orphan receptor $\gamma(\operatorname{ROR} \gamma)$ adult induced knockout mice develop lymphoblastic lymphoma. Autoimmun Rev. 2016;15(11):1062-1070.

17. Han F, Lei H, Lin X, Meng Q, Wang Y, inventor; Glaxo Group Limited, UK, assignee. Preparation of piperazinylmethylphenyl-substituted caboxamides as modulators of the retinoid-related orphan receptor gamma for use in the treatment of autoimmune inflammatory diseases. European patent number WO 201408689. June 12, 2014.

18. Cai Y, et al. Pivotal role of dermal IL-17-producing $\gamma \delta$ T cells in skin inflammation. Immunity. 2011;35(4):596-610.

19. Ivanov II, et al. The orphan nuclear receptor RORgammat directs the differentiation program of proinflammatory IL-17+ $\mathrm{T}$ helper cells. Cell. 2006;126(6):1121-1133.

20. Hirota K, et al. Preferential recruitment of CCR6-expressing Th17 cells to inflamed joints via CCL20 in rheumatoid arthritis and its animal model. J Exp Med. 2007;204(12):2803-2812.

21. Ciofani M, et al. A validated regulatory network for Th17 cell specification. Cell. 2012;151(2):289-303.

22. Nakae S, et al. Antigen-specific T cell sensitization is impaired in IL-17-deficient mice, causing suppression of allergic cellular and humoral responses. Immunity. 2002;17(3):375-387.

23. Okano $\mathrm{H}$, et al. Homozygous deletions and point mutations of the Ikaros gene in gamma-ray-induced mouse thymic lymphomas. Oncogene. 1999;18(48):6677-6683.

24. van den Brandt J, Wang D, Reichardt HM. Resistance of single-positive thymocytes to glucocorticoid-induced apoptosis is mediated by CD28 signaling. Mol Endocrinol. 2004;18(3):687-695.

25. Withers DR, et al. Transient inhibition of ROR- $\gamma \mathrm{t}$ therapeutically limits intestinal inflammation by reducing TH17 cells and preserving group 3 innate lymphoid cells. Nat Med. 2016;22(3):319-323.

26. Pearse G. Histopathology of the thymus. Toxicol Pathol. 2006;34(5):515-547.

27. Martins VC, et al. Cell competition is a tumour suppressor mechanism in the thymus. Nature. 2014;509(7501):465-470.

28. Winandy S, Wu P, Georgopoulos K. A dominant mutation in the Ikaros gene leads to rapid development of leukemia and lymphoma. Cell. 1995;83(2):289-299.

29. Palmer DB. The effect of age on thymic function. Front Immunol. 2013;4:316

30. Boehm T, Swann JB. Thymus involution and regeneration: two sides of the same coin? Nat Rev Immunol. 2013;13(11):831-838 
31. Nindl V, et al. Cooperation of Th1 and Th17 cells determines transition from autoimmune myocarditis to dilated cardiomyopathy. Eur J Immunol. 2012;42(9):2311-2321.

32. Obach RS. Prediction of human clearance of twenty-nine drugs from hepatic microsomal intrinsic clearance data: An examination of in vitro half-life approach and nonspecific binding to microsomes. Drug Metab Dispos. 1999;27(11):1350-1359.

33. Waters NJ, Jones R, Williams G, Sohal B. Validation of a rapid equilibrium dialysis approach for the measurement of plasma protein binding. J Pharm Sci. 2008;97(10):4586-4595.

34. Zhou L, Yang L, Tilton S, Wang J. Development of a high throughput equilibrium solubility assay using miniaturized shakeflask method in early drug discovery. J Pharm Sci. 2007;96(11):3052-3071.

35. Gold DP, et al. Characterization of the immune response to a secondary encephalitogenic epitope of basic protein in Lewis rats II. Biased T cell receptor V beta expression predominates in spinal cord infiltrating T cells. J Immunol. 1992;148(6):1712-1717.

36. Kim G, et al. CDR3 size spectratyping and sequencing of spectratype-derived TCR of spinal cord T cells in autoimmune encephalomyelitis. J Immunol. 1998;160(1):509-513. 